\title{
Wie gut sind angehende und fortgeschrittene Studierende auf das Leben und Arbeiten in der digitalen Welt vorbereitet? Ergebnisse eines Standard Setting-Verfahrens zur Beschreibung von ICT-bezogenen Kompetenzniveaus
}

\author{
Martin Senkbeil (D) J Jan Marten Ihme · Christian Schöber
}

Online publiziert: 26. November 2019

(C) Der/die Autor(en) 2019

Zusammenfassung Bislang gibt es nur wenige empirische Hinweise, zu welchen Anteilen Studierende an deutschen Hochschulen über ausreichende Kompetenzen im Umgang mit digitalen Medien für ihr akademisches und berufliches Fortkommen verfügen. Dieser Frage gehen wir anhand von Sekundäranalysen des Nationalen Bildungspanels (NEPS) nach. Hierfür können die Daten von $N=1911$ angehenden Studierenden sowie von $N=1991$ fortgeschrittenen Studierenden im sechsten Fachsemester ausgewertet werden. Die in NEPS eingesetzten Tests erlauben die Definition von Leistungsbereichen, in denen normativ festgelegte Erwartungen (Mindeststandards) zu verschiedenen Studienzeitpunkten erfüllt werden. Die Analysen zeigen, dass substanzielle Anteile sowohl der angehenden Studierenden (20\%) als auch der fortgeschrittenen Studierenden (52\%) nicht die Mindeststandards erreichen. Weiterhin ergeben sich bei beiden Studierendengruppen zum Teil erhebliche fächergruppen- und geschlechtsbezogene Unterschiede in der Erreichung der Mindeststandards. Die Ergebnisse werden im Hinblick auf die Sicherstellung grundlegender Kompetenzen von Studierenden im Umgang mit digitalen Medien als Kernziel der Hochschulbildung diskutiert.

Zusatzmaterial online Zusätzliche Informationen sind in der Online-Version dieses Artikels (https:// doi.org/10.1007/s11618-019-00914-z) enthalten.

Dr. M. Senkbeil $(\bowtie) \cdot$ Dr. J. M. Ihme · Dr. C. Schöber

IPN - Leibniz-Institut für die Pädagogik der Naturwissenschaften und Mathematik,

Olshausenstraße 62, 24118 Kiel, Deutschland

E-Mail: senkbeil@leibniz-ipn.de

Dr. J. M. Ihme

E-Mail: ihme@ipn.uni-kiel.de

Dr. C. Schöber

E-Mail: schoeber@ifbq.hamburg.de 
Schlüsselwörter Fächergruppen · Digitale Kompetenzen · Mindeststandards · Studierende

\title{
Are first-semester and advanced university students ready for life and work in the digital world? Results of a standard setting method to describe ICT-related proficiency levels
}

\begin{abstract}
There is little empirical evidence available, to what proportions university students possess the necessary ICT (Information and Communication Technology)related competencies (skills) with regard to their academic and professional development. We examine this question by analyzing secondary data of the German National Educational Panel Study (NEPS). We analyzed data from $N=1911$ firstyear students and $N=1991$ advanced students in the sixth semester. The ICT literacy tests used in

NEPS allow the definition of foundational levels that describe the minimum ICT literacy skills a student should have upon entering the first year of university and upon entering upper-division coursework. The results show that substantial proportions of first-year students $(20 \%)$ as well as students in the sixth semester (52\%) do not meet the foundational levels. Furthermore, for both groups of students there are considerable differences in their ICT-related competencies depending on areas of study and gender. The results are discussed with regard to ensuring foundational ICT-related competencies of university students as a core objective of higher education.
\end{abstract}

Keywords Areas of study · Digital literacy · Foundational levels · University students

\section{Einleitung}

Der kompetente Umgang mit Informations- und Kommunikationstechnologien (Information and Communication Technology, ICT Literacy) stellt für Studierende eine unverzichtbare fachübergreifende Kompetenz dar. Sowohl die Zunahme digitaler Lehr- und Lernformate an den Hochschulen als auch die fortschreitende Digitalisierung der Arbeitswelt erfordern, dass große Bereiche des Wissens über die gesamte Lebensspanne weitgehend selbstgesteuert und vornehmlich über digitale Medien anzueignen sind (Acemoglu und Autor 2011). Von den Studierenden wird daher erwartet, dass sie im Umgang mit digitalen Medien zumindest über grundlegende Kompetenzen (z.B. effiziente Informationsrecherche) verfügen (Rott 2014), die $\mathrm{zu}$ einem professionellen Agieren in studienbezogenen und beruflichen Situationen befähigen.

Während international einige Befunde zur ICT Literacy von Studierenden vorliegen (Katz 2007; Blignaut und Els 2010; Smith et al. 2013), wurde diese Domäne bei Studierenden in Deutschland bislang nur ansatzweise untersucht (Heinze et al. 2009; Leichner et al. 2014; Rott 2014). Da die hierzulande durchgeführten Studien nur einzelne Komponenten des Konstrukts (z. B. Informationsrecherche) betrachten, 
kleinere Gelegenheitsstichproben umfassen und teilweise auf Selbsteinschätzungen der Studierenden beruhen, erlauben sie keine validen Aussagen über die Grundgesamtheit der Studierenden in Deutschland. Ungeachtet dieser Einschränkungen deuten die in nationalen wie internationalen Studien berichteten Wissens- und Fertigkeitsdefizite übereinstimmend darauf hin, dass nicht alle Studierenden über die für ihr akademisches und berufliches Fortkommen notwendige ICT Literacy verfügen (Katz 2007; Schulmeister 2009).

Daher wird im vorliegenden Beitrag anhand von Sekundäranalysen des Nationalen Bildungspanels (National Educational Panel Study, NEPS) die wissensbasierte ICT Literacy (im Sinne einer kognitiven Leistungsdisposition) von angehenden und fortgeschrittenen Studierenden an einer repräsentativen Stichprobe untersucht. Hierfür werden die Testwerte beider Gruppen anhand eines Standard-Setting-Verfahrens in Kompetenzniveaus überführt, die eine Bewertung der Kompetenzstände anhand normativ festgelegter Standards ermöglichen (Pant et al. 2010). Anhand der Kompetenzniveaus können die Studierendenanteile bestimmt werden, die zu verschiedenen Studienzeitpunkten über eine ausreichende ICT Literacy im Sinne von Mindeststandards verfügen. Zunächst beschreiben wir das dieser Studie zugrundeliegende Kompetenzstrukturmodell zur ICT Literacy von Studierenden und fassen bislang vorliegende Befunde zur ICT Literacy von Studierenden zusammen. Die Ergebnisse werden abschließend im Hinblick auf die Sicherstellung grundlegender ICTKenntnisse und -Fertigkeiten von Studierenden als Kernziel der Hochschulbildung diskutiert.

\section{Kompetenzstrukturmodelle zur ICT Literacy von Studierenden}

\subsection{Das ICT Literacy Proficiency Model}

Die bislang elaborierteste und am umfangreichsten evaluierte Rahmenkonzeption zur ICT Literacy von Studierenden ist das vom Educational Testing Service (ETS) entwickelte ICT Literacy Proficiency Model (Katz 2007; vgl. auch Sparks et al. 2016 für eine Übersicht verschiedener Konzeptualisierungen). Dieses wurde im Rahmen der National Higher Education ICT Initiative unter Beteiligung nordamerikanischer Hochschulen entwickelt und basiert auf der prominenten Konzeption des International ICT Literacy Panels (ETS 2002) sowie den Information Literacy Competency Standards for Higher Education (ACRL 2000). Danach werden unter ICT Literacy funktionale (d.h. von der Lebens- und Arbeitswelt ausgehende) Wissensbestände und Fertigkeiten im Umgang mit digitalen Medien verstanden, die für eine erfolgreiche Teilhabe an der Gesellschaft und für ein kontinuierliches Weiterlernen bedeutsam sind. ICT Literacy umfasst neben technologischen Kompetenzen (grundlegendes deklaratives und prozedurales Funktionswissen über Programmanwendungen) zusätzlich informationsbezogene Kompetenzen. Diese beinhalteten die Fähigkeit, mit Hilfe digitaler Medien Informationen zu ermitteln, diese kritisch auszuwählen und effektiv für spezifische Zielsetzungen in verschiedenen Anforderungssituationen (z.B. Studium, persönliche Belange) zu nutzen (Katz 2007). ICT Literacy wird als das Zusammenwirken von kognitiven (z.B. schlussfolgerndes Denken, Problemlösen) 
und technologischen Fertigkeiten, d.h. als eindimensionales Konstrukt verstanden und wird folgendermaßen definiert: „ICT literacy is the ability to appropriately use digital technology, communication tools, and/or networks to solve information problems in order to function in an information society. This includes having the ability to use technology as a tool to research, organize, and communicate information“" (Katz 2007, S. 4).

Die Rahmenkonzeption des ICT Literacy Proficiency Model differenziert sieben Prozesskomponenten (siehe Tab. 1 für eine Auflistung und Kurzdarstellung der Prozesse; für eine ausführliche Darstellung: Katz 2007). Diese beschreiben Wissensbestände und Fertigkeiten, über die Studierende für eine erfolgreiche Bewältigung ihres Studiums sowie zukünftiger beruflicher Anforderungen verfügen sollten. Die aus dieser Konzeption abgeleitete Testkonzeption (ETS iskills assessment) berücksichtigt darüber hinaus gebräuchliche Programmanwendungen (z. B. Office-Programme, E-Mail, Webbrowser) sowie verschiedene Kontexte (persönlich, bildungsbezogen). Die Erfassung des Konstrukts erfolgt in Form interaktiver und mehrschrittiger Simulationsaufgaben im Sinne einer verhaltensbasierten ICT Literacy (Katz 2007). Das ETS iskills assessment ermöglicht Hochschulen anhand normativ festgelegter Mindeststandards zu überprüfen, inwieweit ihre Studierenden zu verschiedenen Studienabschnitten (Studienbeginn, Ende des ersten Studienabschnitts) über eine ausreichende ICT Literacy verfügen (Tannenbaum und Katz 2008).

\subsection{ICT Literacy von Studierenden im Nationalen Bildungspanel}

In NEPS wird die ICT Literacy von Studierenden gemäß einem MultikohortenSequenz-Design (Blossfeld et al. 2011) zu verschiedenen Zeitpunkten und in verschiedenen Startkohorten (Starting Cohort, SC) erhoben. Die Erfassung des Konstrukts erfolgt im Gegensatz zum ICT Literacy Proficiency Model (interaktive Simulationsaufgaben) anhand von statischen Aufgaben im Multiple Choice (MC)Format. Aufgrund dieser Limitation im Aufgabenformat erfassen die NEPS-Aufgaben deklarative und prozedurale Wissensbestände des Konstrukts im Sinne einer wissensbasierten ICT Literacy, während die Performanzaufgaben des ICT Literacy Proficiency Model zusätzlich prozedurales Handlungswissen erfassen und somit das Konstrukt im Sinne der Anwendung erworbenen Wissens in realitätsnahen Situationen (verhaltensbasierte ICT Literacy) messen (Senkbeil und Ihme in Vorbereitung; vgl. hierzu auch z. B. Seeber et al. 2010; Blömeke et al. 2015). Entsprechend decken die NEPS-Tests eine geringere Konstruktbreite ab und erfassen ein Teilkonstrukt von ICT Literacy (vgl. Senkbeil und Ihme in Vorbereitung; Sparks et al. 2016).

Mit Ausnahme dieser Limitation in der Konstruktbreite orientiert sich die Rahmenkonzeption von ICT Literacy im nationalen Bildungspanel in hohem Maße am ICT Literacy Proficiency Models bzw. an der Konzeption des ICT Literacy Panels. Konkret gründet die Konzeptualisierung in NEPS auf derselben Definition und differenziert weitgehend identische Prozesskomponenten und Programmanwendungen in einem Strukturmodell (siehe online verfügbares Zusatzmaterial, Anhang A oder Senkbeil et al. 2013). Entsprechend weisen beide Konzeptualisierungen eine hohe Übereinstimmung auf, wie die Gegenüberstellung in Tab. 1 zeigt. Wie beim ICT Literacy Proficiency Model ist ICT Literacy in NEPS als eindimensionales Konstrukt 
Tab. 1 Differenzierung der ICT Literacy in den Rahmenkonzeptionen des ICT Literacy Proficiency Model und des Nationalen Bildungspanels (NEPS)

\begin{tabular}{|c|c|}
\hline ICT Literacy Proficiency Model & NEPS ICT Literacy \\
\hline \multicolumn{2}{|l|}{ Prozesskomponenten $(P)$} \\
\hline $\begin{array}{l}\text { P1: Define: Grundlegendes Verständnis über } \\
\text { Hardware und Software; Formulierung informa- } \\
\text { tionsbezogener Fragestellungen }\end{array}$ & $\begin{array}{l}\text { P1: Anwenden/Verstehen: Grundkenntnisse über } \\
\text { Software, Kenntnis grundlegender Operationen, um } \\
\text { Informationen zu ermitteln und abzurufen }\end{array}$ \\
\hline \multicolumn{2}{|l|}{$\begin{array}{l}\text { P2: Access: Ermitteln und Abrufen von Informa- } \\
\text { tionen }\end{array}$} \\
\hline $\begin{array}{l}\text { P3: Create: Dateien bearbeiten, anwenden oder } \\
\text { erstellen }\end{array}$ & $\begin{array}{l}\text { P2: Erzeugen: Fähigkeit, Dateien zu bearbeiten } \\
\text { oder zu erstellen }\end{array}$ \\
\hline $\begin{array}{l}\text { P4: Manage: Informationen organisieren, z. B. } \\
\text { miteinander vergleichen oder sortieren }\end{array}$ & $\begin{array}{l}\text { P3: Suchen/Organisieren: Informationen effizient } \\
\text { ermitteln, Informationen nach spezifischen Kriteri- } \\
\text { en vergleichen oder sortieren }\end{array}$ \\
\hline $\begin{array}{l}\text { P5: Integrate: Informationen verschiedener Da- } \\
\text { tenquellen zusammenfassen, vergleichen, trans- } \\
\text { formieren }\end{array}$ & - \\
\hline $\begin{array}{l}\text { P6: Evaluate: Informationen bewerten, Entschei- } \\
\text { dungen treffen }\end{array}$ & $\begin{array}{l}\text { P4: Bewerten: Informationen bewerten, Entschei- } \\
\text { dungen treffen }\end{array}$ \\
\hline $\begin{array}{l}\text { P7: Communicate: Informationen zielgruppen- } \\
\text { gerecht kommunizieren (z. B. E-Mails schreiben, } \\
\text { Präsentationen informativ gestalten) }\end{array}$ & $\begin{array}{l}\text { P5: Kommunizieren: Informationen angemessen } \\
\text { und verständlich kommunizieren (z. B. E-Mails } \\
\text { schreiben, informative Grafiken erstellen) }\end{array}$ \\
\hline \multicolumn{2}{|l|}{ Programmanwendungen } \\
\hline & Betriebssystem \\
\hline Textverarbeitung & Textverarbeitung \\
\hline \multirow[t]{2}{*}{ Tabellenkalkulation } & Tabellenkalkulation \\
\hline & Präsentationsprogramme \\
\hline E-Mail & E-Mail/Chats/Internetforen \\
\hline Internetgestützte Suchmaschinen/Datenbanken & Internetgestützte Suchmaschinen/Datenbanken \\
\hline \multicolumn{2}{|l|}{ Kontexte } \\
\hline Bildungsbezogen & Bildungsbezogen \\
\hline Persönlich & Persönlich \\
\hline
\end{tabular}

konzeptualisiert, bei dem die Aufschlüsselung nach verschiedenen Dimensionen dazu dient, die zentralen kognitiven Prozesse, Programmanwendungen und Kontexte zu definieren (vgl. Senkbeil et al. 2013; Sparks et al. 2016).

Zusammenfassend lässt sich somit bilanzieren, dass die Erfassung von ICT Literacy in NEPS bei Studierenden konzeptuell sowie in der Testoperationalisierung an das ICT Literacy Proficiency Model anschlussfähig ist. Sie erlaubt weitergehend eine Überprüfung der Kompetenzen in Form kriterienbezogener Rückmeldeformate, welche die Leistungsverteilung auf kategorial gestuften Kompetenzniveaus abbilden (Pant et al. 2010).

\section{Internationale und nationale Befunde zur ICT Literacy}

Mit dem ETS iskills assessment durchgeführte und auf großen Stichproben basierende Evaluationen $(N>6000$; Katz 2007) in Nordamerika zeigen, dass mehr als 
die Hälfte der angehenden wie auch der fortgeschrittenen Studierenden den jeweils festgelegten Mindeststandard verfehlen (Tannenbaum und Katz 2008). Insbesondere bei informationsbezogenen Problemstellungen wie z.B. der effizienten Informationssuche im Internet, dem Bewerten von Informationen oder dem adressatengerechten Erstellen von Informationsprodukten (z.B. Texte, Präsentationen) weisen die untersuchten Studierenden mehrheitlich gravierende Defizite auf (Wallace und Clariana 2005; Katz 2007). Weiterhin ergeben sich erwartungsgemäß eine höhere ICT Literacy für fortgeschrittene Studierende im Vergleich zu Studienanfängern sowie Kompetenzunterschiede in Abhängigkeit vom Studienfach (Troboy et al. 2009; Teske und Etheridge 2010). Zu ähnlich ernüchternden Befunden, wonach die Studierenden mehrheitlich nicht den normativ festgelegten Mindeststandard erreichen, kommen Evaluationsstudien aus Kanada und Südafrika, die jedoch auf vergleichsweise kleinen Stichproben $(N \approx 100)$ basieren (Blignaut und Els 2010; Smith et al. 2013). Weiter gestützt werden diese Befunde durch eine Reihe von Studienergebnissen, die im Rahmen des Information Problem Solving-Paradigmas ermittelt wurden (Walraven et al. 2009). Bei verschiedenen Aufgabenstellungen zeigte sich, dass Studierende häufig Probleme haben, adäquate Suchbegriffe zu formulieren, und ermittelte Informationen oftmals nur anhand oberflächlicher Kriterien hinsichtlich ihrer Glaubwürdigkeit und Nützlichkeit einschätzen (Walraven et al. 2009; Nikolopoulou und Gialamas 2011; Goldman et al. 2012). Mit diesen Befunden korrespondierend fehlt es Hochschulabsolventen bei Berufseintritt aber häufig an grundlegenden digitalen Kompetenzen (z. B. effiziente Informationsrecherche, Umgang mit Office- oder E-Mail-Programmen) wie auch an realistischen Vorstellungen darüber, welche digitalen Kompetenzen die jeweilige Berufsausübung erfordert (Littlejohn et al. 2012; Raish und Rimland 2016).

Für Deutschland liegen bislang nur wenige empirische Befunde zur ICT Literacy von Studierenden vor. Studien zu einzelnen Komponenten, z. B. der Informationskompetenz, deuten - analog zu den internationalen Befunden - auf zum Teil erhebliche Wissensdefizite von Studierenden hin, z. B. bei der Suche nach wissenschaftlichen Informationen und deren Bewertung (z.B. Heinze 2008; Schmidt-Hertha und Rott 2014; Mayer et al. 2016). Aufgrund ihrer methodischen Beschränkungen (z. B. kleine Gelegenheitsstichproben, Berücksichtigung weniger Studienfächer, zum Teil Erfassung selbsteingeschätzter Fähigkeiten) erlauben sie aber keine generalisierenden Aussagen.

Aufgrund fehlender Studien werden häufig Daten zur freizeit- und studienbezogenen Nutzung digitaler Medien von Studierenden zur Einschätzung ihrer ICT Literacy herangezogen. So zeigt sich, dass Studierende häufig nur unzureichend in der Lage sind, ihre ICT-bezogenen Wissensbestände und Fertigkeiten auf hochschulrelevante Lerninhalte zu übertragen (Schulmeister 2009) und in der Mehrzahl ein begrenztes Verständnis von innovativen und digitalen Lehr-Lernszenarien (z. B. Blended Learning, kollaborative Arbeitsformen) haben. Die studienbezogene Nutzung digitaler Medien fällt bei vielen Studierenden entsprechend konservativ aus und beschränkt sich weitgehend auf klassische digitale Medien wie PDF-Dokumente, E-Mail und Präsentationsprogramme (Persike und Friedrich 2016). Da sie digitale Medien im privaten Kontext zudem vergleichsweise eingeschränkt, d. h. größtenteils zur Unterhaltung und zum sozialen Austausch nutzen, werden bei Studierenden un- 
zureichende digitale Kompetenzen im Hinblick auf ihr akademisches Fortkommen vermutet (Schulmeister 2010).

Darüber hinaus sind studienfachspezifische Unterschiede in der ICT Literacy zu erwarten. Da Personen mit hohen kognitiven Grundfähigkeiten und aus akademischem Elternhäusern eher prestigeträchtige Studienfächer (z. B. Medizin, Jura) wählen und sich seltener für sprach- und kulturwissenschaftliche Fächer entscheiden (Becker et al. 2010), sind bereits zu Studienbeginn fachspezifische Kompetenzunterschiede zuungunsten der Sprach- und Kulturwissenschaften anzunehmen. Darüber hinaus variiert die Nutzung digitaler Lehr- und Lernformate stark über die Studienfächer (Kleimann et al. 2008). Während in den Sprach- und Kulturwissenschaften vornehmlich klassische digitale Medien Anwendung finden, werden in den Ingenieurswissenschaften (z. B. Informatik) häufig zusätzlich interaktive Werkzeuge (z.B. Webkonferenzen) eingesetzt, was sich in höherem Maße als kompetenzförderlich auswirken sollte (Hofhues 2016). Zu Studienbeginn bestehende Kompetenzunterschiede könnten sich demnach während des Studiums noch vergrößern.

Weiterhin werden geschlechtsspezifische Disparitäten in der ICT Literacy bei Studierenden zuungunsten des weiblichen Geschlechts angenommen. Trotz inkonsistenter Befundlage werden für Studentinnen geringere Kompetenzen vermutet, da sie im Vergleich zu Studenten eine höhere ICT-bezogene Ängstlichkeit sowie ein geringeres ICT-bezogenes Selbstkonzept aufweisen und damit einhergehend digitale Lehr- und Lernangebote eher meiden (Huang et al. 2013).

\section{Fragestellungen und Hypothesen}

Zentrales Ziel dieses Beitrags ist die Untersuchung der ICT Literacy von angehenden und fortgeschrittenen Studierenden (sechstes Fachsemester) für verschiedene Fächergruppen und in Abhängigkeit vom Geschlecht. Die in den NEPS-Tests erzielten Testwerte werden anhand eines Standard-Setting-Verfahrens in Kompetenzniveaus unterteilt, die eine kriterienbezogene Bewertung der Leistungen ermöglichen. Wie beim ETS iskills Assessment (Tannenbaum und Katz 2008) sollen die Kompetenzniveaus die minimalen Wissensbestände und Fertigkeiten in Form von Mindeststandards beschreiben, die Studierende zu verschiedenen Zeitpunkten für ihr akademisches und berufliches Fortkommen benötigen.

Da alle nachfolgend aufgeführten Hypothesen auf der Annahme eines eindimensionalen ICT Literacy-Konstrukts basieren, wird vorab geprüft (Hypothese 0, H0), inwieweit diese Voraussetzung erfüllt ist, d.h. ob die Prozesskomponenten ausreichend hoch korrelieren und über alle Prozesskomponenten vergleichbare und homogene Kompetenzstände für die untersuchten Gruppen (Studienfachgruppen, Geschlecht) vorliegen. Basierend auf den referierten Überlegungen werden folgende inhaltliche Fragestellungen und Hypothesen untersucht:

Fragestellung 1 Über welche Kompetenzstände und Kompetenzniveaus verfügen angehende und fortgeschrittene Studierende? 
- Hypothese 1 (H1): Da Studierende digitale Medien sowohl für studienbezogene als auch private Zwecke nutzen (z. B. Kleimann et al. 2008), weisen fortgeschrittene Studierende eine höhere ICT Literacy auf als angehende Studierende.

- Hypothese 2 (H2): Gemäß der referierten nationalen und internationalen Befundlage (z. B. Heinze 2008; Tannenbaum und Katz 2008) vermuten wir bei den angehenden Studierenden (H2a) und auch bei den fortgeschrittenen Studierenden (H2b) substanzielle Anteile ( $\geq 20 \%$ ), die den jeweiligen Mindeststandard nicht erreichen.

Fragestellung 2 Ergeben sich Kompetenzunterschiede in Abhängigkeit vom Studienfach und Geschlecht?

- Hypothese 3 (H3): Aufgrund der referierten Befunde zu herkunftsbedingten Disparitäten in der Studienfachwahl (Becker et al. 2010) und fachspezifischen Unterschieden in der Nutzung digitaler Medien (z. B. Hofhues 2016) erwarten wir für angehende Studierende (H3a) und fortgeschrittene Studierende (H3b) fachgruppenspezifische Unterschiede in der ICT Literacy. Dabei werden zumindest teilweise Effektstärken von $d \geq 0,50$ erwartet, z. B. zwischen den Ingenieurswissenschaften und den Sprach- und Kulturwissenschaften.

- Hypothese 4 (H4): Daran gekoppelt sollten zwischen den Fachgruppen Unterschiede in den Studierendenanteilen auftreten, die den jeweiligen Mindeststandard erreichen (H4a: angehende Studierende; H4b: fortgeschrittene Studierende).

- Hypothesen 5 und 6 (H5, H6): Angesichts der inkonsistenten Befundlage (Huang et al. 2013) werden für Studenten allenfalls geringe Kompetenzvorteile (mit Effektstärken von $d \leq 0,30)$ gegenüber Studentinnen vermutet (H5a: angehende Studierende, H5b: fortgeschrittene Studierende). Entsprechend sollten Studienanfänger (H6a) und fortgeschrittene Studenten (H6b) zu etwas größeren Anteilen auf den höheren Kompetenzniveaus vertreten sein.

Fragestellung 3 Lassen sich Effekte der Eingangsselektivität auf die ICT Literacy von angehenden und fortgeschrittenen Studierenden ermitteln?

- Hypothese 7 (H7): Da kognitive Grundfähigkeiten stark mit ICT Literacy assoziiert (Senkbeil et al. 2013) sind und bei der Studienfachwahl eine substanzielle Rolle spielen (Becker et al. 2010) sowie die Geschlechter sehr unterschiedlich in den Fächergruppen vertreten sind (siehe Abschnitt 5.1), wird abschließend der Frage nachgegangen, inwieweit fachspezifische Kompetenzunterschiede durch diese Eingangsmerkmale vermittelt werden. Hier ist die Erwartung, dass nach Kontrolle von kognitiven Grundfähigkeiten und Geschlecht im Sinne einer Mediation die Kompetenzunterschiede abnehmen (H7a: angehende Studierende, H7b: fortgeschrittene Studierende).

\section{Methode}

Die empirische Datengrundlage für die vorliegende Arbeit bildeten Erhebungen in NEPS. Die Anlage und Durchführung von NEPS ist detailliert in Blossfeld et al. 
Tab. 2 Verteilung der angehenden und fortgeschrittenen Studierenden auf die Fächergruppen

\begin{tabular}{|c|c|c|c|c|c|c|}
\hline \multirow[t]{2}{*}{ Fächergruppe $^{\mathrm{a}}$} & \multicolumn{3}{|c|}{ Angehende Studierende } & \multicolumn{3}{|c|}{ Fortgeschrittene Studierende } \\
\hline & Absolut & $\begin{array}{l}\%(\mathrm{Un}- \\
\text { gew.) }\end{array}$ & $\begin{array}{l}\% \\
\text { (Gew.) }\end{array}$ & Absolut & $\begin{array}{l}\% \text { (Un- } \\
\text { gew.) }\end{array}$ & $\begin{array}{l}\% \\
\text { (Gew.) }\end{array}$ \\
\hline $\begin{array}{l}\text { Rechts-, Wirtschafts- und } \\
\text { Sozialwissenschaften }\end{array}$ & 596 & 31,2 & 33,4 & 446 & 22,4 & 33,4 \\
\hline $\begin{array}{l}\text { Humanmedizin, Gesundheits- } \\
\text { wissenschaften }\end{array}$ & 96 & 5,0 & 5,6 & 128 & 6,4 & 4,4 \\
\hline $\begin{array}{l}\text { Ingenieurswissenschaften } \\
\text { (inklusive Informatik) }\end{array}$ & 349 & 18,3 & 20,5 & 205 & 10,3 & 21,0 \\
\hline $\begin{array}{l}\text { Mathematik, Naturwissen- } \\
\text { schaften }\end{array}$ & 416 & 21,8 & 18,4 & 443 & 22,3 & 17,0 \\
\hline $\begin{array}{l}\text { Sprach- und Kulturwissen- } \\
\text { schaften }\end{array}$ & 353 & 18,5 & 16,1 & 668 & 33,6 & 17,3 \\
\hline Sonstige Fächergruppen ${ }^{\mathrm{b}}$ & 101 & 5,2 & 6,0 & 101 & 5,0 & 6,9 \\
\hline Gesamt & 1911 & 100 & 100 & 1991 & 100 & 100 \\
\hline
\end{tabular}

ungew. ungewichtet; gew. gewichtet

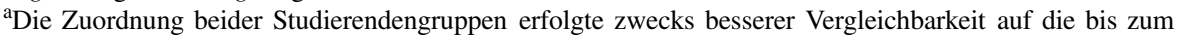
Wintersemester 2015/16 gültige Fächersystematik des Statistischen Bundesamts

bSonstige Fächergruppen: Sport, Agrar-, Forst- und Ernährungswissenschaften, Veterinärmedizin sowie Kunst und Kunstwissenschaften

(2011) beschrieben. Wir beschränken uns daher im Folgenden auf die für die vorliegende Arbeit wesentlichen Informationen. Diese umfassen die Beschreibung der Stichproben, der ICT Literacy-Tests sowie der Linking-Studie, mit der die ICT Literacy-Tests beider Studierendengruppen auf einer gemeinsamen Metrik skaliert wurden. Abschließend wird das Standard-Setting-Verfahren zur Generierung von kategorialen Kompetenzniveaus erläutert.

\subsection{Stichproben}

Für die Beantwortung der Fragestellungen berücksichtigen wir die Daten derjenigen Studierenden, für die ICT Literacy-Testwerte sowie valide Angaben zum gewählten Studienfach vorliegen. Der ICT Literacy-Test für die angehenden Studierenden wurde in der Schule am Ende der 12. Jahrgangsstufe in 2013 (SC4, Welle 7) durchgeführt, also ein Jahr vor Studienbeginn. Die Stichprobe umfasst alle Schülerinnen und Schüler, die im darauffolgenden Jahr ein Studium aufgenommen haben $(N=1911$; weiblich: 53,9\%). Die Testung der fortgeschrittenen Studierenden erfolgte $2013 \mathrm{im}$ 6. Fachsemester (SC5, Welle 5). Von dieser Stichprobe können $N=1991$ (weiblich: 60,6\%) Studierende in die Analysen aufgenommen werden. Um repräsentative Ergebnisse zu erhalten, wurden die Daten für die Analysen entsprechend den Populationsverteilungen gewichtet (siehe Tab. 2).

Dabei zeigt sich erwartungskonform, dass Studentinnen seltener ein ingenieurswissenschaftliches (Anteil SC4: 25\%, Anteil SC5: 22\%) und ein mathematischnaturwissenschaftliches Fach (Anteil jeweils knapp unter 40\%) wählen. Bei allen anderen Fächergruppen bildeten sie die Mehrheit (zwischen 55\% in den Rechts-, 


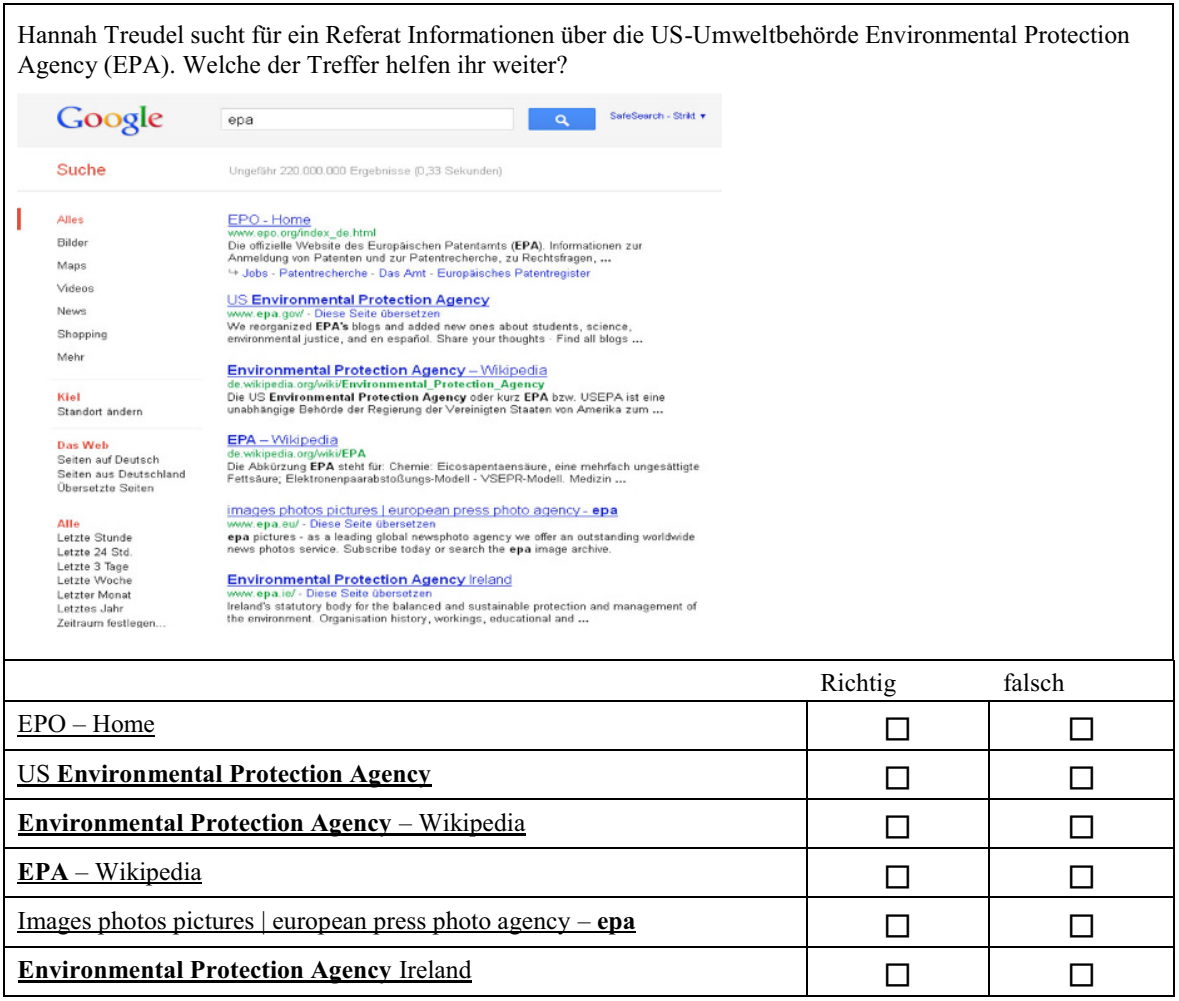

Abb. 1 Beispielitem zur Proesskomponente Bewertung (Programmanwendung: Internet); Kompetenzniveau Basic (476 Punkte)

Wirtschafts- und Sozialwissenschaften und $75 \%$ in den Sprach- und Kulturwissenschaften).

\subsection{Instrumente}

ICT Literacy-Tests. Beide Tests, die bei den Studierenden eingesetzt wurden, umfassen bei einer Bearbeitungszeit von $30 \mathrm{~min} 31$ bzw. 29 MC-Aufgaben (vgl. Senkbeil und Ihme 2017). Die Aufgaben enthalten authentische Problemstellungen und erfassen, ob die Testpersonen angemessen mit bestimmten Aufgabenstellungen umgehen können. Für eine möglichst realitätsnahe Gestaltung werden in den Aufgabenstimulus Screenshots integriert (z. B. von einem Internet-Browser) und als Distraktoren häufig realistische Antwortalternativen in Form von Schaltflächen oder Menüs vorgegeben (siehe Abb. 1 und 2 für Beispielitems). Durch in NEPS implementierte Linking-Studien (siehe auch Abschnitt 5.3) können die Kompetenztests über verschiedene Kohorten und Messzeitpunkte gemeinsam skaliert werden (Pohl und Carstensen 2013). 


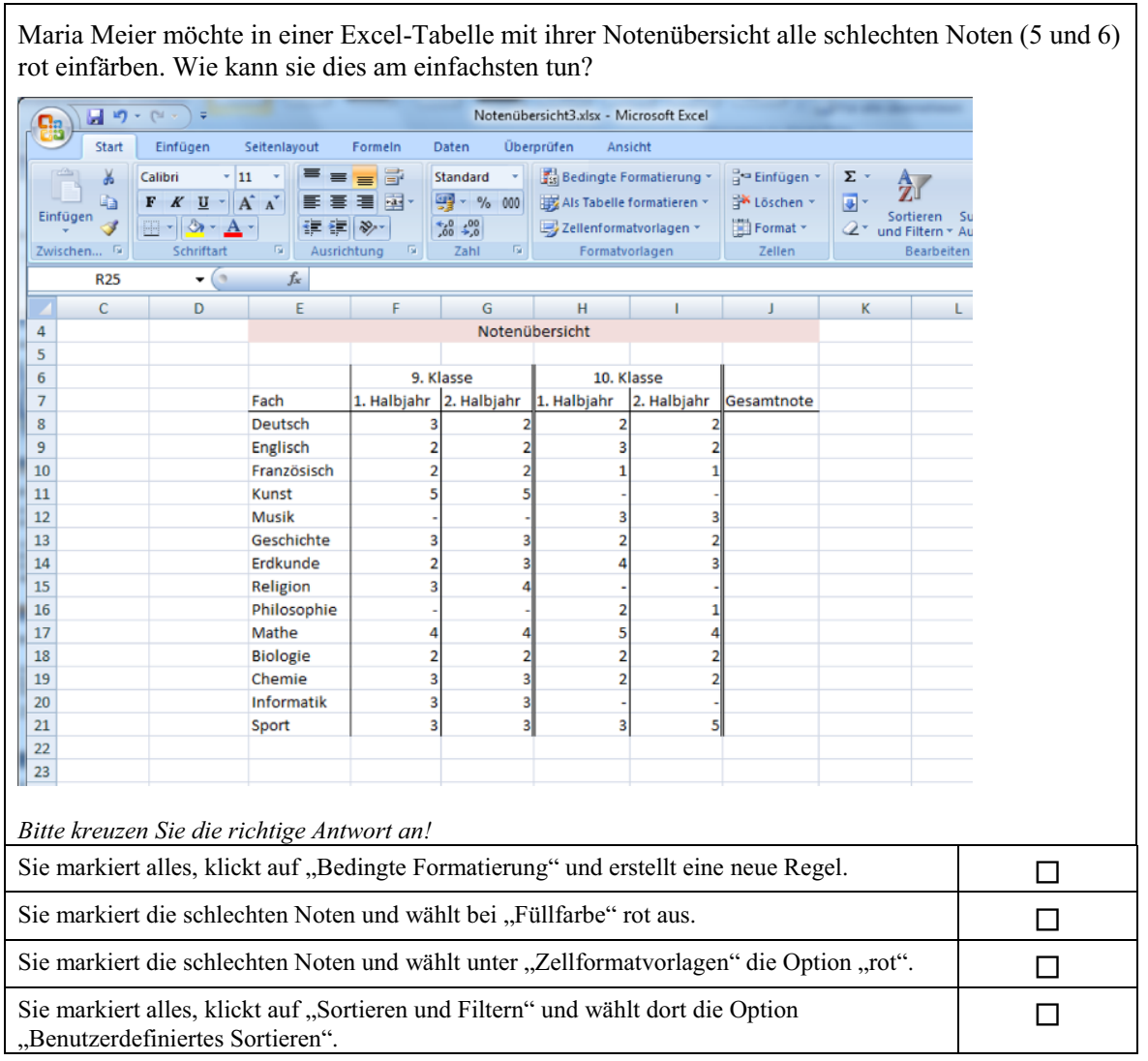

Abb. 2 Beispielitem zur Proesskomponente Suchen und Organisieren (Programmanwendung: Tabellenkalkulation); Kompetenzniveau Proficient (663 Punkte)

Kognitive Grundfähigkeiten Zur Erfassung der kognitiven Grundfähigkeiten wird in NEPS ein sprachfreier Test zum schlussfolgernden Denken (NEPS-MAT, Haberkorn und Pohl 2013) eingesetzt. Dieser ist in der Tradition klassischer Reasoning-Tests als Matrizentest angelegt, umfasst 12 Aufgaben bei einer Bearbeitungszeit von $9 \mathrm{~min}$ und besitzt eine ausreichende Reliabilität (Cronbachs $\alpha=0,73$ ). Der individuelle Leistungswert ergibt sich durch den Summenscore über alle 12 Items.

\subsection{Linking-Studie}

Zur Verknüpfung der beiden ICT Literacy-Tests standen Daten einer im Herbst 2013 durchgeführten Linking-Studie in NEPS zur Verfügung. In dieser bearbeiteten insgesamt $N=394$ Schülerinnen und Schüler der zwölften Klasse aus vier Bundesländern sowohl den Test für Klasse 12 (SC4) als auch den Test für die fortgeschrittenen Studierenden (SC5). Die insgesamt 60 Items beider Tests wurden für die Gesamtstichprobe (Linking-Stichprobe, Klasse 12 und fortgeschrittene Studierende) mittels eines eindimensionalen Raschmodells in ConQuest (Wu et al. 2007) unter Verwen- 
dung eines Hintergrundmodells (Fächergruppe, Geschlecht, kognitive Grundfähigkeiten) gemeinsam skaliert. Die auf diese Weise ermittelten Kompetenzwerte (fünf Plausible Values, $P V s$ ) wurden in der gewichteten Stichprobe der fortgeschrittenen Studierend en auf einen Mittelwert von $M=500$ und $S D=100$ transformiert. Die EAP/PV-Reliabilität des Gesamttests beträgt 0,72 und ist somit als ausreichend anzusehen (Rost 2004).

\subsection{Standard Setting-Verfahren}

Um Kompetenzniveaus für beide Studierendengruppen zu generieren, wurde ein Standard-Setting-Verfahren (Pant et al. 2010) auf Grundlage der gemeinsamen Skalierung aller Items durchgeführt. In dem Standard-Setting bestimmte ein Expertenpanel $^{1}$ aus den Bereichen ICT Literacy und digitales Lehren und Lernen an der Hochschule diejenigen Wissensbestände und Fertigkeiten, über die eine Person für ein bestimmtes Kompetenzniveau verfügen muss, und legte Grenzwerte (so genannte Cut-Scores) zur Trennung der Kompetenzniveaus fest. Hierfür wurde die Item-Descriptor-Matching-Methode angewendet, d.h. die Anforderungen jeder Aufgabe wurden mit vorab erstellten Rohversionen der Kompetenzniveaubeschreibungen (Ferrara und Lewis 2012) abgeglichen. Dem Verfahren liegt ein iteratives Verfahren zugrunde, bei dem sich das Expertenpanel erst individuell und anschließend als Gruppe auf konsensuelle Cut-Scores verständigt. In einem zweiten Schritt wurden die Kompetenzniveaubeschreibungen modifiziert und ausgeschärft, indem die Aufgaben dahingehend analysiert wurden, welche kognitiven Operationen zur erfolgreichen Lösung benötigt werden.

Als Ergebnis dieses Vorgehens wurden die Aufgaben den Kompetenzniveaus $\mathrm{Ba}$ sic und Proficient zugeordnet. Die beiden Kompetenzniveaus wurden anhand der fünf Prozesskomponenten der NEPS ICT Literacy-Rahmenkonzeption beschrieben (siehe Tab. 3). Das Kompetenzniveau Basic definiert ein digitales Grundbildungsniveau, das für die Aufnahme eines Studiums notwendig ist und den Erwerb fortgeschrittener, d.h. für das Studium und den späteren Beruf benötigte Kompetenzen ermöglicht. Das Kompetenzniveau Proficient beschreibt ein erweitertes Grundbildungsniveau, welches Studierende im Verlauf ihres Studiums im Hinblick auf spätere berufliche Anforderungen erwerben sollten. Die Kompetenzniveaus repräsentieren damit Mindeststandards, die ein Studierender zu verschiedenen Studienzeitpunkten erfüllen sollte. Darüber hinaus wurde ein Cut-Score bestimmt, der die notwendigen Wissensbestände und Fertigkeiten beschreibt, damit ein Studierender überhaupt als digital grundgebildet gelten kann. Studierende unterhalb dieses Cut-Scores (Kompetenzniveau Below Basic) verfügen nur über rudimentäre Wissensbestände und Fertigkeiten, besitzen beispielsweise keine ausreichenden Kenntnisse im Umgang mit Office-Programmen und haben grundlegende Probleme bei der Identifizierung, Bewertung und Weiterverarbeitung ermittelter Informationen.

\footnotetext{
1 Das Expertenpanel setzte sich aus folgenden Personen zusammen: Julia Gerick, Frank Goldhammer, Michael Leucht, Andreas Mühling, Tom Rosman, Jan Vahrenhold und Johannes Zylka.
} 
Tab. 3 Ergebnisse des Standard-Settings: Wertebereiche und Beschreibungen der Kompetenzniveaus in NEPS ICT Literacy

\begin{tabular}{|c|c|c|}
\hline \multicolumn{2}{|c|}{ Kompetenzniveau } & \multirow{2}{*}{$\begin{array}{l}\text { Benötigte ICT-bezogene Wissensbestände und Fertigkeiten (Prozesskompo- } \\
\text { nenten) } \\
\text { Grundlegende Kenntnisse im Umgang mit gängigen Office-Programmen (z. B. } \\
\text { Textverarbeitung, Tabellenkalkulation): z. B. einfache Formatierungen durch- } \\
\text { führen, Zellen auswählen, Dateien in einem anderen Format speichern }\end{array}$} \\
\hline $\begin{array}{l}\text { Basic } \\
319-506 \\
\text { Punkte }\end{array}$ & P1: & \\
\hline & P2: & $\begin{array}{l}\text { Informationen in einfacher Form weiterverarbeiten: z. B. Auswahl einer Dar- } \\
\text { stellungsform auf Basis zweckdienlicher Kriterien }\end{array}$ \\
\hline & P3: & $\begin{array}{l}\text { Informationen (z. B. in Dokumenten, im Internet) identifizieren und abrufen: } \\
\text { z. B. Durchsuchen von Dokumenten nach bestimmten Begriffen, Nutzung } \\
\text { von Suchmaschinen, Sortieren und Filtern von Informationen nach einzelnen } \\
\text { Kriterien }\end{array}$ \\
\hline & P4: & $\begin{array}{l}\text { Informationen nach einzelnen, explizit vorgegebenem Kriterium bewerten: z. B. } \\
\text { Relevanz, Verständlichkeit, Glaubwürdigkeit }\end{array}$ \\
\hline & P5: & $\begin{array}{l}\text { Grundlegende Kenntnisse und Fertigkeiten für die Nutzung digitaler Kommu- } \\
\text { nikationswerkzeuge (z. B. E-Mail, soziale Netzwerke): z. B. Unterscheidung } \\
\text { zwischen öffentlichen und privaten Informationen, verschiedene Arten von } \\
\text { Adressaten beim E-Mail-Verkehr berücksichtigen }\end{array}$ \\
\hline \multirow[t]{5}{*}{$\begin{array}{l}\text { Proficient } \\
\geq 507 \\
\text { Punkte }\end{array}$} & P1: & $\begin{array}{l}\text { Weiterführende Kenntnisse im Umgang mit gängigen Office-Programmen (z. B. } \\
\text { Textverarbeitung, Tabellenkalkulation): z. B. Arbeiten mit Formatvorlagen, } \\
\text { automatisiertes Erstellen eines Inhaltsverzeichnisses }\end{array}$ \\
\hline & P2: & $\begin{array}{l}\text { Informationen in komplexerer Form weiterverarbeiten: z. B. Zusammenführen } \\
\text { mehrerer Informationsquellen in ein neues Informationsprodukt, Ableitung von } \\
\text { Kenngrößen aus vorhandenen Daten }\end{array}$ \\
\hline & P3: & $\begin{array}{l}\text { Weiterführende Kenntnisse und Fertigkeiten für Identifikation und Abruf von } \\
\text { Informationen: Anwendung komplexerer Strategien zum Auffinden gesuchter } \\
\text { Informationen, Sortieren und Filtern von Informationen nach mehreren Kriteri- } \\
\text { en }\end{array}$ \\
\hline & P4: & $\begin{array}{l}\text { Informationen nach mehreren, implizit vorgegebenen Kriterien bewerten: z. B. } \\
\text { Relevanz, Verständlichkeit, Glaubwürdigkeit }\end{array}$ \\
\hline & P5: & $\begin{array}{l}\text { Weiterführende Kenntnisse und Fertigkeiten für die Nutzung digitaler Kom- } \\
\text { munikationswerkzeuge (z. B. E-Mail, soziale Netzwerke): z. B. Erkennen von } \\
\text { Phishing-Nachrichten, Verwaltung von Nutzerkonten }\end{array}$ \\
\hline
\end{tabular}

\subsection{Analysen}

Für die Analysen zur Beantwortung der Hypothesen wurde die Statistiksoftware SPSS 24 verwendet. Zwecks Prüfung der Vorab-Hypothese (H0) wurde für den Gesamttest mit 60 Items in ConQuest ein fünfdimensionales Raschmodell gemäß den Prozesskomponenten (Anwenden/Verstehen: 9 Items, Erzeugen: 15 Items; Kommunizieren: 10 Items; Bewerten: 13 Items; Suchen/Organisieren: 13 Items) berechnet. Als Personenparameterschätzer wurden Weighted Likelihood Estimates (WLEs) bestimmt. Zur Bestimmung der Kompetenzen (H1, H3, H5) wurden zunächst die mittleren Kompetenzwerte unter Berücksichtigung der Populationsgewichte für die jeweilige Studierendengruppe berechnet. Da die Mittelwerte und anderen statistischen Kennwerte auf fünf PVs beruhen, wurden alle Analysen fünfmal durchgeführt und die Ergebnisse gemäß den Vorschlägen von Rubin (Rubin's Rules 1987; vgl. auch Rutkowski et al. 2010) gemittelt. Für die Mittelwerte der Fächergruppen wurde inferenzstatistisch anhand der Standardfehler geprüft, ob sie signifikant vom 
jeweiligen Gesamtmittelwert abweichen. In einem weiteren Schritt wurden mit den Schwellenwerten (Cut-Scores) aus dem Standard-Setting-Verfahren die Verteilungen auf den Kompetenzstufen berechnet (H2, H4, H6). Diese Analysen (Mittelwerte, Kompetenzniveauverteilungen) wurden für die jeweilige Gesamtstichprobe sowie differenziert nach Fächergruppen und Geschlecht durchgeführt. Zur Klärung der Frage, inwieweit Kompetenzunterschiede zwischen den Fächergruppen durch die Eingangsvoraussetzungen vermittelt werden, wurden für beide Studierendengruppen multiple Regressionsanalysen durchgeführt (H7).

\section{Ergebnisse}

Zunächst wird geprüft, inwieweit die grundlegende Annahme eines eindimensionalen und homogenen ICT Literacy-Konstrukts gerechtfertigt ist. Für die Prozesskomponenten ergeben sich gemäß der eindimensionalen Testkonstruktion substanzielle Interkorrelationen zwischen 0,80 (Anwenden/Verstehen und Bewerten) und 0,88 ( $\mathrm{Su}$ chen/Organisieren und Bewerten). Für die verschiedenen Fächergruppen zeigen sich über alle Prozesskomponenten für die verschiedenen Fächergruppen weitgehend homogene, d. h. nahezu durchgehend überschneidungsfreie Kompetenzprofile (z-standardisierte WLEs als Personenparameterschätzer; siehe Abb. 3). Nur die Fachgruppe der Humanmedizin und Gesundheitswissenschaften weist ein inhomogenes Kompetenzprofil auf: Vergleichsweise geringen Kompetenzwerten in den Komponenten Anwenden/Verstehen, Erzeugen und Kommunizieren stehen hohe Kompetenzwerte in den Komponenten Bewerten und Suchen/Organisieren gegenüber. Davon abgesehen belegen die jeweiligen Fächergruppen in allen Prozesskomponenten die gleiche Rangposition (bis auf eine Vertauschung in der Rangfolge bei der Prozesskomponente Suchen/Organisieren), wenngleich die Höhe der Kompetenzunterschiede zwischen den Fächergruppen über die Prozesskomponenten variiert.

In Bezug auf das Geschlecht zeigt sich konsistent bei allen Prozesskomponenten ein Kompetenzvorteil für die Studenten, der ebenfalls in der Höhe leicht variiert (Abb. 4).

Aufgrund der erwarteten, weitgehend homogenen Kompetenzprofile erscheint die Überprüfung der inhaltlichen Hypothesen auf Grundlage des Gesamttests gerechtfertigt. Das abweichende Kompetenzprofil für die Fachgruppe der Humanmedizin und Gesundheitswissenschaften sowie die variierenden Kompetenzunterschiede zwischen den Fächergruppen über die Prozesskomponenten werden bei der Diskussion berücksichtigt.

\subsection{ICT Literacy bei angehenden und fortgeschrittenen Studierenden}

Wie Tab. 4 ausweist, verfügen fortgeschrittene Studierende erwartungsgemäß über eine höhere ICT Literacy als angehende Studierende (H1a). Der Unterschied von 100 Punkten (501 vs. 401 Punkte) entspricht einer Effektstärke von $d=1,00$, was auf einen erheblichen Kompetenzzuwachs in der ersten Studienphase hinweist. Ebenfalls hypothesenkonform ergeben sich bei angehenden Studierenden (H3a) und bei fortgeschrittenen Studierenden (H3b) zum Teil beträchtliche Unterschiede in der 


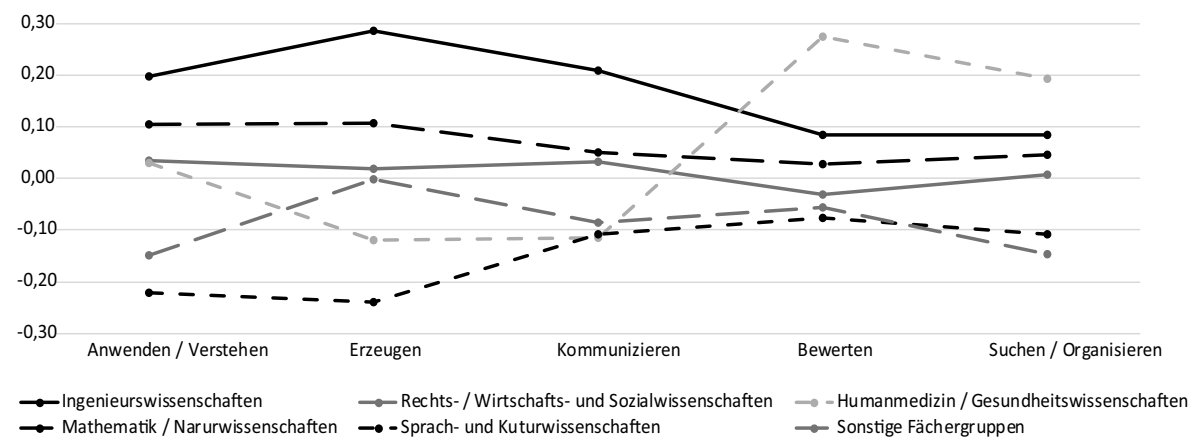

Abb. 3 Mittlere Kompetenzwerte (z-standardisierte WLEs) für die Prozesskomponenten, differenziert nach Fächergruppen. Anmerkung: WLE Weighted Likelihood Estimate (Personenparameterschätzer)

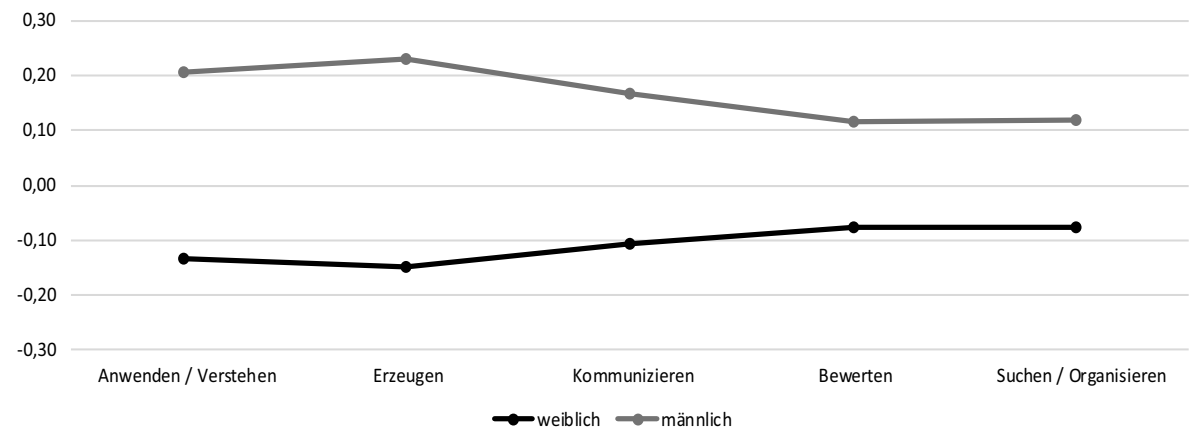

Abb. 4 Mittlere Kompetenzwerte (z-standardisierte WLEs) für die Prozesskomponenten, differenziert nach Geschlecht. Anmerkung: WLE Weighted Likelihood Estimate (Personenparameterschätzer)

ICT Literacy zwischen den Fächergruppen. Bei den angehenden Studierenden weisen die Studierenden der Sprach- und Kulturwissenschaften mit 368 Punkten die geringste geringere ICT Literacy (368 Punkte) auf. Sie liegen zwischen 32 und 53 Punkten hinter den Studierenden den Rechts-, Wirtschafts- und Sozialwissenschaften $(d=0,36)$, der Medizin $(d=0,42)$, den Ingenieurswissenschaften $(d=0,58)$ und den mathematisch-naturwissenschaftlichen Fächern $(d=0,58)$. Ähnlich ungünstig schneiden Studierende der sonstigen Fächergruppen (Sport, Agrar-, Forst- und Ernährungswissenschaften, Veterinärmedizin, Kunst und Kunstwissenschaften) mit 372 Punkten ab. Deren Kompetenzrückstände zu den übrigen Fächergruppen fallen nahezu identisch aus $(0,39 \leq d \leq 0,61)$.

Weiterhin erzielen männliche Studienanfänger deutlich höhere Kompetenzwerte als die weiblichen Studienanfänger (H5a). Der Unterschied fällt mit 52 Punkten $(d=0,57)$ größer aus als erwartet und ist nicht auf die unterschiedliche Verteilung der Geschlechter auf die Fächergruppen zurückzuführen, d. h. es ergeben sich bei einer zweifaktoriellen Varianzanalyse mit den Faktoren Fächergruppe und Geschlecht keine signifikanten Interaktionseffekte.

Diese Unterschiede zeigen sich in sehr ähnlicher Form, d.h. mit identischen Rangfolgen bei den fortgeschrittenen Studierenden. Im Vergleich zu den angehen- 
Tab. 4 ICT Literacy der angehenden und fortgeschrittenen Studierenden: Mittelwerte $(M)$, Standardabweichungen $(S D)$ und Standardfehler $(S E)$

\begin{tabular}{llllllll}
\hline & Fächergruppe & \multicolumn{3}{c}{ Angehende Studierende } & \multicolumn{3}{c}{ Fortgeschrittene Studierende } \\
& & $M$ & $S D$ & $S E$ & $M$ & $S D$ & $S E$ \\
\hline 1 & $\begin{array}{l}\text { Rechts-, Wirtschafts- und } \\
\text { Sozialwissenschaften }\end{array}$ & 400 & 96 & 3,95 & 502 & 117 & 5,56 \\
& & & & & & \\
2 & $\begin{array}{l}\text { Humanmedizin, Gesund- } \\
\text { heitswissenschaften }\end{array}$ & 410 & 96 & 9,78 & 496 & 83 & 7,36 \\
3 & Ingenieurswissenschaften & $421^{\mathrm{a}}$ & 101 & 5,40 & $537^{\mathrm{a}}$ & 143 & 10,00 \\
4 & Mathematik, Naturwis- & $418^{\mathrm{a}}$ & 87 & 4,27 & $517^{\mathrm{a}}$ & 84 & 3,99 \\
& senschaften & & & & & & \\
5 & Sprach- und Kulturwis- & $368^{\mathrm{b}}$ & 84 & 4,46 & $461^{\mathrm{b}}$ & 66 & 2,56 \\
& senschaften & & & & & & \\
6 & Sonstige Fächergruppen & $372^{\mathrm{b}}$ & 98 & 9,78 & $457^{\mathrm{b}}$ & 105 & 10,48 \\
- & Eta & 0,046 & - & - & 0,076 & - & - \\
7 & Weiblich & $375^{\mathrm{b}}$ & 86 & 2,71 & $467^{\mathrm{b}}$ & 81 & 2,22 \\
8 & Männlich $^{\mathrm{b}}$ & $427^{\mathrm{a}}$ & 98 & 3,40 & $535^{\mathrm{a}}$ & 115 & 4,48 \\
- & Eta & 0,070 & - & - & 0,116 & - & - \\
- & Gesamt $^{\mathrm{b}}$ & 401 & 95 & 2,18 & 501 & 100 & 2,23 \\
\hline
\end{tabular}

${ }^{a}$ Mittelwert liegt signifikant über dem Gesamtmittelwert $(p<0,05)$;

${ }^{\mathrm{b}}$ Mittelwert liegt signifikant unter dem Gesamtmittelwert $(p<0,05)$

den Studierenden fallen die Unterschiede zwischen den Fächergruppen und den Geschlechtern prägnanter aus, wie an den größeren Varianzanteilen, die durch die Fächergruppenzugehörigkeit (Eta ${ }^{2}: 7,6 \%$ vs. 4,6\%) und das Geschlecht (Eta ${ }^{2}: 11,6 \%$ vs. 7,0\%) aufgeklärt werden können, deutlich wird. Entsprechend fallen die Kompetenzrückstände der Studierenden der Sprach- und Kulturwissenschaften (35 bis 75 Punkte; $0,45 \leq d \leq 0,75$ ) und der sonstigen Fächergruppen (39 bis 79 Punkte; $0,41 \leq d \leq 0,64)$ noch größer aus. Die Studierenden der Ingenieurswissenschaften erzielen mit 537 Punkten wiederum das beste Ergebnis. Ebenso fallen die Kompetenzvorteile der männlichen Studierenden im sechsten Fachsemester mit 68 Punkten $(d=0.69)$ höher aus als bei Studienbeginn (H5b) und sind wiederum nicht durch Interaktionseffekte zwischen Fächergruppe und Geschlecht aufklärbar. Zusammengefasst stützen die Ergebnisse unsere Erwartung, wonach Studierende in Bezug auf ihre ICT Literacy keine homogene Population darstellen. Auffällig sind jedoch die unerwartet starken geschlechtsbezogenen Kompetenzunterschiede. Im Folgenden wird berichtet, zu welcher Verteilung auf die Kompetenzniveaus die berichteten mittleren Unterschiede führen.

\subsection{Kompetenzniveaus der angehenden und fortgeschrittenen Studierenden}

Wie im Methodenteil erläutert, definieren die vom Expertenpanel festgelegten Kompetenzniveaus jeweilige Mindeststandards für die angehenden Studierenden (Basic) und die fortgeschrittenen Studierenden (Proficient). Studierende, die nicht das Kompetenzniveau Basic erreichen, verfügen über keine ausreichende digitale Grundbildung, die für die Aufnahme eines Studiums notwendig ist. Wie Tab. 5 zeigt, ergeben sich bei beiden Studierendengruppen sowohl für die Fächergruppen als auch für das 
Tab. 5 Verteilung der angehenden und fortgeschrittenen Studierenden auf die vom Expertenpanel definierten Kompetenzniveaus, getrennt nach Fächergruppen und Geschlecht (Angaben in Prozent)

\begin{tabular}{|c|c|c|c|c|c|c|}
\hline \multirow[b]{2}{*}{ Fächergruppe } & \multicolumn{3}{|c|}{ Angehende Studierende } & \multicolumn{3}{|c|}{ Fortgeschrittene Studierende } \\
\hline & $\begin{array}{l}\text { Below } \\
\text { Basic }\end{array}$ & Basic & Proficient & $\begin{array}{l}\text { Below } \\
\text { Basic }\end{array}$ & Basic & Proficient \\
\hline $\begin{array}{l}\text { Rechts-, Wirtschafts- und } \\
\text { Sozialwissenschaften }\end{array}$ & 19,7 & 67,2 & 13,1 & 2,3 & 49,5 & 48,2 \\
\hline $\begin{array}{l}\text { Humanmedizin, Gesundheits- } \\
\text { wissenschaften }\end{array}$ & 17,2 & 68,5 & 14,3 & 4,3 & 50,0 & 45,7 \\
\hline Ingenieurswissenschaften & 14,3 & 67,0 & 18,8 & 1,8 & 35,6 & 62,6 \\
\hline $\begin{array}{l}\text { Mathematik, Naturwissen- } \\
\text { schaften }\end{array}$ & 15,4 & 67,2 & 17,3 & 1,9 & 44,1 & 54,0 \\
\hline $\begin{array}{l}\text { Sprach- und Kulturwissen- } \\
\text { schaften }\end{array}$ & 29,7 & 63,9 & 6,4 & 6,2 & 61,8 & 32,0 \\
\hline Sonstige Fächergruppen & 31,2 & 63,5 & 5,3 & 6,3 & 63,1 & 30,6 \\
\hline Weiblich & 26,7 & 65,9 & 7,4 & 5,4 & 60,3 & 34,3 \\
\hline Männlich & 13,0 & 67,0 & 19,9 & 1,0 & 37,5 & 61,5 \\
\hline Gesamt & 20,0 & 66,5 & 13,5 & 3,2 & 48,7 & 48,1 \\
\hline
\end{tabular}

Ergebnisse mit SPSS gemittelt über jeweils fünf Plausible Values

Geschlecht starke Unterschiede in der Erreichung der jeweiligen Mindeststandards (H4, H6).

Unserer Hypothese entsprechend (H2a) unterschreiten insgesamt $20 \%$ der angehenden Studierenden den festgelegten Mindeststandard (Kompetenzniveau Basic). Bei den Studierenden der sprach- und kulturwissenschaftlichen Fächer sowie der sonstigen Fächergruppen beträgt der Anteil sogar mehr als ein Viertel (30\% bzw. $31 \%$ ). Bei den andern Fächergruppen fallen diese Anteile deutlich geringer aus und betragen zwischen 14\% (Ingenieurswissenschaften) und 20\% (Rechts-, Wirtschaftsund Sozialwissenschaften; H4a). In Bezug auf das Geschlecht zeigt sich, dass $27 \%$ der weiblichen Studienanfänger den Mindeststandard nicht erreichen. Der Anteil ist mehr als doppelt so hoch wie bei den männlichen Studienanfängern (13\%; H6a).

Im sechsten Fachsemester erreichen $48 \%$ der Studierenden das Kompetenzniveau Proficient. Trotz offensichtlich erheblicher Kompetenzzuwächse in der ersten Studienphase (siehe H1a) erreicht also etwas mehr als die Hälfte der Studierenden (noch) nicht den normativ festgelegten Standard, was deutlich über dem vorab vermuteten Prozentsatz liegt (H2b). Am schlechtesten schneiden Studierende der Sprachund Kulturwissenschaften und der sonstigen Fächergruppen ab. Von diesen erreicht weniger als ein Drittel (32\% bzw. 31\%) den Mindeststandard (H4b). Immerhin etwa $6 \%$ der Studierenden dieser Fächergruppen unterschreiten auch noch zu diesem Zeitpunkt den Mindeststandard der angehenden Studierenden.

Die höchsten Studierendenanteile auf Kompetenzniveau Proficient finden sich bei den Ingenieurswissenschaften $(63 \%)$ und den mathematisch-naturwissenschaftlichen Fächern (54\%). Weiterhin verfügen nahezu alle Studierende dieser beiden Fächergruppen - wie auch der Rechts-, Wirtschafts- und Sozialwissenschaften zumindest über ein ausreichendes Maß digitaler Grundbildung (Kompetenzniveau Basic). 
Tab. 6 ICT Literacy der angehenden Studierenden in Abhängigkeit der kognitiven Grundfähigkeiten und dem Geschlecht (unstandardisierte Regressionskoeffiezienten (B) und Standardfehler (SE))

\begin{tabular}{|c|c|c|c|c|c|c|}
\hline & \multicolumn{2}{|l|}{ Modell 1} & \multicolumn{2}{|l|}{ Modell 2} & \multicolumn{2}{|l|}{ Modell 3} \\
\hline & $\mathrm{B}$ & $(S E)$ & $\mathrm{B}$ & $(S E)$ & $\mathrm{B}$ & $(S E)$ \\
\hline Intercept & 420,8 & $(4,7)$ & 429,7 & $(4,7)$ & 428,3 & $(4,6)$ \\
\hline Geschlecht $^{\mathrm{a}}$ & - & - & $-44,3 * * *$ & $(4,4)$ & $-47,1 * * *$ & $(4,3)$ \\
\hline Kognitive Grundfähigkeiten ${ }^{b}$ & - & - & - & - & $27,2 * * *$ & $(2,0)$ \\
\hline \multicolumn{7}{|l|}{ Fächergruppen ${ }^{\mathrm{c}}$} \\
\hline $\begin{array}{l}\text { Rechts-, Wirtschafts- und } \\
\text { Sozialwissenschaften }\end{array}$ & $-20,7 * * *$ & $(6,1)$ & $-7,4$ & $(6,0)$ & 1,7 & $(5,8)$ \\
\hline $\begin{array}{l}\text { Humanmedizin, Gesundheits- } \\
\text { wissenschaften }\end{array}$ & $-10,7$ & $(10,3)$ & 9,2 & $(10,1)$ & 11,1 & $(9,7)$ \\
\hline $\begin{array}{l}\text { Mathematik, Naturwissen- } \\
\text { schaften }\end{array}$ & $-2,9$ & $(7,0)$ & 3,2 & $(6,7)$ & 6,0 & $(6,5)$ \\
\hline $\begin{array}{l}\text { Sprach- und Kulturwissen- } \\
\text { schaften }\end{array}$ & $-53,2 * * *$ & $(7,2)$ & $-31,7 * * *$ & $(7,2)$ & $-23,2 * *$ & $(7,1)$ \\
\hline Sonstige Fächergruppen & $-48,6^{* * *}$ & $(10,0)$ & $-44,2 * * *$ & $(9,7)$ & $-35,8^{* *}$ & $(9,4)$ \\
\hline$R^{2}$ & 0,05 & - & 0,09 & - & 0,18 & - \\
\hline
\end{tabular}

aReferenzgruppe: männlich

$\mathrm{b}_{\mathrm{z} \text {-standardisiert }(M=0, S D=1)}$

${ }^{\mathrm{c}}$ Referenzgruppe: Ingenieurswissenschaften; multiple Regressionsanalysen mit SPSS (gemittelt über jeweils fünf Plausible Values); $* p<0,05, * * p>0,01, * * * p>0,001$

Große Unterschiede sind auch hinsichtlich des Geschlechts festzustellen (H6b). Deutlich mehr als die Hälfte der fortgeschrittenen Studenten (62\%) erreicht den Mindeststandard Proficient, dies trifft jedoch nur auf ein Drittel der fortgeschrittenen Studentinnen zu (34\%). Während die Studenten im sechsten Semester nahezu flächendeckend zumindest über eine digitale Grundbildung verfügen, erreichen mehr als 5\% der fortgeschrittenen Studentinnen auch zu diesem Zeitpunkt nicht dieses Niveau.

\subsection{Kompetenzunterschiede unter Kontrolle von kognitiven Grundfähigkeiten und Geschlecht}

In multiplen Regressionsanalysen wird geprüft, inwieweit die Fachgruppenunterschiede in der ICT Literacy durch bestimmte die Eingangsmerkmale kognitive Grundfähigkeiten und Geschlecht vermittelt werden. Die Ergebnisse der dazu spezifizierten Regressionsmodelle mit den entsprechenden Regressionskoeffizienten sind in Tab. 6 für die angehenden Studierenden und Tab. 7 für die fortgeschrittenen Studierenden dargestellt.

Modell 1 gibt jeweils noch einmal die Kompetenzunterschiede zwischen den Fächergruppen für die jeweilige Studierendengruppe wieder. Die unstandardisierten Regressionskoeffizienten (B) entsprechen den Mittelwertdifferenzen zur Referenzgruppe (Ingenieurswissenschaften) in Tab. 4. In Modell 2 wird zusätzlich das Geschlecht und in Modell 3 darüber hinaus die kognitiven Grundfähigkeiten aufgenommen. Durch die Berücksichtigung des Geschlechts in Modell 2 erhöhen sich die Anteile aufgeklärter Varianz bereits deutlich (angehende Studierende: $\Delta R^{2}=0,04$; 
Tab. 7 ICT Literacy der fortgeschrittenen Studierenden in Abhängigkeit der kognitiven Grundfähigkeiten und dem Geschlecht (unstandardisierte Regressionskoeffiezienten (B) und Standardfehler (SE))

\begin{tabular}{|c|c|c|c|c|c|c|}
\hline & \multicolumn{2}{|l|}{ Modell 1} & \multicolumn{2}{|l|}{ Modell 2} & \multicolumn{2}{|l|}{ Modell 3} \\
\hline & $\mathrm{B}$ & $(S E)$ & $\mathrm{B}$ & $(S E)$ & $\mathrm{B}$ & $(S E)$ \\
\hline Intercept & 536,7 & $(4,7)$ & 545,1 & $(4,6)$ & 540,7 & $(4,6)$ \\
\hline Geschlecht $^{\mathrm{a}}$ & - & - & $-57,7 * * *$ & $(4,4)$ & $-49,8 * * *$ & $(2,0)$ \\
\hline Kognitive Grundfähigkeiten ${ }^{\mathrm{b}}$ & - & - & - & - & $27,0 * * *$ & $(2,0)$ \\
\hline \multicolumn{7}{|l|}{ Fächergruppen $^{c}$} \\
\hline $\begin{array}{l}\text { Rechts-, Wirtschafts- und } \\
\text { Sozialwissenschaften }\end{array}$ & $-33,8 * * *$ & $(6,0)$ & $-14,9$ & $(5,9)$ & $-8,0$ & $(5,7)$ \\
\hline $\begin{array}{l}\text { Humanmedizin, Gesundheits- } \\
\text { wissenschaften }\end{array}$ & $-40,6^{* *}$ & $(11,2)$ & $-14,7$ & $(11,0)$ & $-11,8$ & $(10,5)$ \\
\hline $\begin{array}{l}\text { Mathematik, Naturwissen- } \\
\text { schaften }\end{array}$ & $-18,6$ & $(7,0)$ & $-8,4$ & $(6,8)$ & $-9,9$ & $(6,5)$ \\
\hline $\begin{array}{l}\text { Sprach- und Kulturwissen- } \\
\text { schaften }\end{array}$ & $-75,6 * * *$ & $(7,0)$ & $-44,3 * * *$ & $(7,0)$ & $-36,3^{* *}$ & $(6,8)$ \\
\hline Sonstige Fächergruppen & $-79,5 * * *$ & $(9,6)$ & $-61,2 * * *$ & $(9,2)$ & $-54,3 * *$ & $(8,9)$ \\
\hline$R^{2}$ & 0,07 & - & 0,15 & - & 0,22 & - \\
\hline
\end{tabular}

aReferenzgruppe: männlich

$\mathrm{b}_{\mathrm{Z} \text {-standardisiert }(M=0, S D=1)}$

${ }^{c}$ Referenzgruppe: Ingenieurswissenschaften

multiple Regressionsanalysen mit SPSS (gemittelt über jeweils fünf Plausible Values); * $p<0,05$, $* * p>0,01, * * * p>0,001$

fortgeschrittene Studierende: $\Delta R^{2}=0,08$ ), und die Aufnahme der kognitiven Grundfähigkeiten in Modell 3 führt zu einer weiteren Zunahme aufgeklärter Varianz (angehende Studierende: $\Delta R^{2}=0,09$; fortgeschrittene Studierende: $\Delta R^{2}=0,07$ ).

Die jeweiligen Veränderungen in den Regressionskoeffizienten zwischen Modell 1 (Ausgangsmodell) und Modell 3 (finales Modell) weisen auf die vermuteten Mediationseffekte hin (H7a, H7b), da sich bei beiden Studierendengruppen die Abstände zur Referenzgruppe der Ingenieurswissenschaften erheblich verringern (angehende Studierende: zwischen 9 und 30 Punkten; fortgeschrittene Studierende: zwischen 9 und 39 Punkten). Bei den angehenden Studierenden ergeben sich für einige Fächergruppen (z.B. Mathematik und Naturwissenschaften) sogar geringe, aber nicht signifikante Kompetenzvorteile. Zusammengefasst verschwinden bei den Fächergruppen Rechts-, Wirtschafts- und Sozialwissenschaften, Mathematik und Naturwissenschaften sowie Humanmedizin und Gesundheitswissenschaften die vormals signifikanten Mittelwertdifferenzen im Vergleich zu den Ingenieurswissenschaften. Bei den anderen Fächergruppen (Sprach- und Kulturwissenschaften, sonstige Fächergruppen) bleiben hingegen signifikante Kompetenzrückstände bestehen. Während diese bei den angehenden Studierenden angesichts der gewählten Metrik $(S D=100)$ vergleichsweise gering ausfallen, entsprechen sie bei den fortgeschrittenen Studierenden zumindest mittelgroßen Effekten. Im Einklang mit den Hypothesen (H7a, H7b) lässt sich somit zusammenfassen, dass die Kompetenzunterschiede zwischen den Fächergruppen zumindest teilweise durch die Eingangsselektivität (kognitive Grundfähigkeiten, Geschlecht) vermittelt, aber nicht vollständig durch diese aufgeklärt werden. 


\section{Diskussion}

Mit dem vorliegenden Beitrag präsentieren wir Befunde zur bislang noch offenen Fragestellung, in welchem Umfang Studierende in Deutschland über die für ihr akademisches und berufliches Fortkommen notwendige ICT Literacy verfügen. Da es hierzu bislang an belastbaren und verallgemeinerbaren Befunden fehlte (Rott 2014), gingen wir dieser Frage anhand einer Sekundäranalyse von Daten des Nationalen Bildungspanels nach. Darüber hinaus konnte die ICT Literacy mittels eines StandardSetting-Verfahrens auf kategorial gestuften Kompetenzskalen (Kompetenzniveaus) abgebildet werden. Diese erlauben eine Bewertung der Leistungen zu verschiedenen Studienabschnitten (Studienbeginn, gegen Ende des ersten Studienabschnitts) anhand normativ festgelegter Erwartungen.

Im Folgenden diskutieren wir die Befunde der beiden Studierendengruppen zunächst anhand der Kompetenzniveaus. Anschließend widmen wir uns den Kompetenzdifferenzen zwischen den untersuchten Gruppen (Fächergruppen, Geschlecht) und berücksichtigen hierbei Limitationen der vorliegenden Studie sowie offene Fragestellungen.

\subsection{Verteilung auf die Kompetenzniveaus}

Erwartungskonform - und im Einklang mit Ergebnissen nordamerikanischer Evaluationsstudien (Tannenbaum und Katz 2008; Smith et al. 2013) - weisen substanzielle Anteile von angehenden und auch vergleichsweise erfahrenen Studierenden (sechstes Fachsemester) beträchtliche Defizite in ihrer ICT Literacy auf. Etwa ein Fünftel der angehenden Studierenden verfügt über kein hinreichendes Maß digitaler Grundbildung, welches für die Aufnahme eines Studiums als notwendig angesehen wird. Angesichts dieser nur rudimentären Wissensbestände und Fertigkeiten sind für diese Studierenden Schwierigkeiten im Studium und im Beruf nicht auszuschließen (Rott 2014), Gegen Ende des sechsten Fachsemesters verfügen nahezu alle Studierenden $(97 \%)$ über ein digitales Grundbildungsniveau, welches zwar den Erwerb fortgeschrittener Kompetenzen ermöglicht, aber eigentlich bereits zu Studienbeginn erwartet wird.

Das zu diesem Zeitpunkt normativ erwartete Kompetenzniveau Proficient erreicht jedoch nur knapp die Hälfte der fortgeschrittenen Studierenden (48\%). Berücksichtigt man, dass typische Berufsfelder von Studierenden zunehmend fortgeschrittene Kenntnisse mit Office-Anwendungen (z.B. Tabellenkalkulation) sowie elaborierte Lösungsstrategien zu komplexen informationsbezogenen Problemstellungen (z.B. Abruf, Selektion und Bewertung spezifischer Informationen nach multiplen Kriterien) erfordern (z. B. Rott 2014; Schmidt-Hertha und Rott 2014), werden Kompetenzen auf Grundbildungsniveau hierfür aller Wahrscheinlichkeit nach nicht ausreichend sein.

Diese Befunde korrespondieren mit zentralen Befunden der internationalen Schulleistungsstudie International Computer and Information Literacy Study (ICILS) 2013 und ergänzen diese (Bos et al. 2014). Dort zeigte sich neben einem generell nur mittelmäßigem Abschneiden der Achtklässlerinnen und Achtklässler in Deutschland auch für Gymnasien, die einen Großteil der zukünftig Studierenden stellen, dass 
nur knapp die Hälfte (48\%) der Schülerinnen und Schüler ein Kompetenzniveau erreicht, welches eine selbstbestimmte und reflektierte Teilhabe in einer digitalisierten Welt erwarten lässt (z.B. selbstständiges Lösen informationsbezogener Problemstellungen, Erstellen inhaltlich gehaltvoller Informationsprodukte; Bos et al. 2014; Eickelmann 2017). Auch wenn die Studien NEPS und ICILS 2013 methodisch und in ihrer Anlage nicht direkt miteinander vergleichbar sind, deuten die Ergebnisse darauf hin, dass sich die in Klasse 8 ermittelten Kompetenzdefizite substanzieller Anteile von Schülerinnen und Schülern bis ins Studium fortsetzen und in der weiteren Schullaufbahn nicht mehr (vollständig) kompensiert werden.

Der hohe Anteil digital illiterater angehender Studierender unterstreicht die Dringlichkeit, mit der die von der Kultusminsterkonferenz (KMK) verabschiedete Strategie „Bildung in der digitalen Welt“ in den Schulen umzusetzen ist. Danach sind aus z.B. ICILS 2013 abgeleitete Kernbereiche digitaler Bildung möglichst frühzeitig in der Schule zu vermitteln, um ein digitales Grundbildungsniveau sicherzustellen, welches den Erwerb fortgeschrittener Kompetenzen (z. B. im Studium) überhaupt erst ermöglicht (KMK 2016; vgl. Eickelmann 2017). Für die Hochschulen fehlen bislang vergleichbar elaborierte Digitalisierungsstrategien. Vielmehr wird vergleichsweise häufig auf noch ungenutzte Potenziale hingewiesen, z. B. die unzureichende technische und didaktische Qualifizierung und Weiterbildung der Hochschullehrenden - als den treibenden Kräften für die Etablierung digitaler Lehr- und Lernformate -und fehlende Anreizsysteme (z. B. Lehrfreisemester, Berufungsverfahren) für diese (z.B. Hochschulforum Digitalisierung 2016; Pensel und Hofhues 2017).

\subsection{Kompetenzniveaus nach Fächergruppen und Geschlecht}

Besonders hohe Anteile von Studierenden unterhalb des jeweiligen Mindeststandards konnten wir in Sprach- und Kulturwissenschaften sowie in den sonstigen Fächergruppen (Sport, Agrar-, Forst- und Ernährungswissenschaften, Veterinärmedizin, Kunst) identifizieren. Nahezu ein Drittel der Studierenden in diesen Fächergruppen beginnt ein Studium ohne ausreichende digitale Grundbildung. Dies trifft sogar nach sechs Fachsemestern auf immerhin noch 6\% der Studierenden zu. Für diese Gruppe sind neben Schwierigkeiten in Studium und Beruf auch Probleme im Hinblick auf die private Lebensführung (z. B. Online-Banking, E-Government) zu befürchten (van Deursen und van Dijk 2009). Umgekehrt erreicht nur knapp ein Drittel das zu diesem Zeitpunkt erwartete Kompetenzniveau Proficient. Dies deutet auf fehlende Lerngelegenheiten in diesen Fächergruppen über den bisherigen Studienverlauf hin. Aber selbst in den leistungsstärksten Fächergruppen, den Ingenieurswissenschaften und den mathematisch-naturwissenschaftlichen Fächern, erreichen bei weitem nicht alle Studierenden die jeweiligen Mindeststandards (angehende Studierende: $\approx 15 \%$; fortgeschrittene Studierende: 37 bzw. 46\%). Die vergleichsweise hohen Anteile über alle Fächergruppen unterstreichen die Dringlichkeit, an den Hochschulen Rahmenbedingungen zu schaffen, die eine Förderung der ICT Literacy von Studierenden - in mehr oder weniger allen Studiengängen - ermöglichen (z. B. Hochschulform Digitalisierung 2016).

Eine ungünstige Befundlage zeigt sich auch für Studentinnen. Ihr Anteil an angehenden Studierenden ohne ausreichende digitale Grundbildung ist mit nahezu $27 \%$ 
mehr als doppelt so hoch wie bei Studenten. Auch ihnen scheint es während des Studiums - unabhängig vom Studienfach - nicht zu gelingen, diese Kompetenzdefizite gegenüber den Studenten aufzuholen, da zwei Drittel der Studentinnen nach sechs Fachsemestern nicht das normativ erwartete Kompetenzniveau Proficient erreichen (männliche Studierende: 62\%).

\subsection{Kompetenzunterschiede und Ausblick}

Die fachgruppenspezifischen Unterschiede in den Kompetenzniveaus entsprechen erwartungskonform zum Teil mittleren bis starken Effektstärken. Dies gilt vornehmlich für die Sprach- und Kulturwissenschaften sowie die sonstigen Fächergruppen im Vergleich mit den Ingenieurswissenschaften und den mathematisch-naturwissenschaftlichen Fächern (Studienbeginn: $0,49 \leq d \leq 0,58$; sechstes Fachsemester: $0,63 \leq d \leq 0,75)$. Dieses Ergebnis korrespondiert mit Befunden zur digitalen Mediennutzung, wonach Studierende technologiebezogener und mathematisch-naturwissenschaftlicher Studienfächer eine größere Affinität gegenüber digitalen Medien aufweisen als Studierende der Sprach- und Kulturwissenschaften und diese insgesamt und insbesondere für informationsbezogene Zwecke häufiger nutzen (Kleimann et al. 2008; Persike und Friedrich 2016). Auch unter Kontrolle differenzieller Eingangsmerkmale (kognitive Grundfähigkeiten, Geschlecht) verbleiben immer noch signifikante und in der Größenordnung substanzielle Kompetenzrückstände, insbesondere bei den fortgeschrittenen Studierenden (36 bzw. 54 Kompetenzpunkte). Da in der vorliegenden Studie nur zwei Selektionsvariablen berücksichtigt wurden, ist der Frage nach der Eingangsselektivität in zukünftigen Studien genauer nachgehen.

Die den eigentlichen, inhaltlichen Hypothesen vorgeschaltete Analyse zu den Prozesskomponenten weist für nahezu alle Fächergruppen auf relativ homogene, d.h. gleichförmige Kompetenzprofile, aber auf keine ausgeprägten Stärken oder Schwächen einzelner Fächergruppen hin. So ist die Rangfolge der Fächergruppen über alle Prozesskomponenten identisch, was zum Teil auch Folge des Einsatzes vergleichsweise homogener Items im Sinne einer eindimensionalen Testkonzeption ist. Die einzige Ausnahme bildet die Fachgruppe der Humanmedizin und Gesundheitswissenschaften mit ausgeprägten Stärken beim Bewerten sowie Suchen und Organisieren digital bereitgestellter Informationen. Diese Stärken sind möglicherweise in einer fachbezogenen Tradition bei der Nutzung digitaler Lehr-Lernformate begründet. Mögliche, in den Fächertraditionen begründete Stärken und Schwächen in bestimmten Komponenten der ICT Literacy können anhand eines Grundbildungstests wie dem vorliegenden jedoch nicht oder nur schwer identifiziert werden und bedürfen gesonderter Testentwicklungen (z. B. zum kompetenten Umgang mit Kommunikationswerkzeugen, vgl. z. B. Hofhues 2016). Weiterhin ist genauer zu klären, inwieweit die Kompetenzunterschiede z.B. auf unterschiedliche Muster und Präferenzen der digitalen Mediennutzung im Jugendalter (z. B. instrumentelle Nutzungsmuster bei technologie-affinen vs. hedonistische Nutzungsmuster bei sprachlich-kulturell interessierten Jugendlichen; vgl. Senkbeil 2017) oder soziale Ungleichheiten im Laufe der Mediensozialisation (Hollingworth et al. 2011) zurückgeführt werden können.

Die ähnlich hohen und damit erwartungswidrigen Kompetenzrückstände von mehr als einer halben Standardabweichung der Studentinnen gegenüber den Stu- 
denten kontrastieren hingegen z. B. mit Befunden aus ICILS 2013. Dort haben die Jungen in Klasse 8 auf dem Gymnasium einen geringen, aber nicht signifikanten Kompetenzvorsprung $(d=0,08)$ gegenüber den Mädchen (Bos et al. 2014). Inwieweit möglicherweise geschlechtsbezogene Selektionseffekte bei der Entscheidung für oder gegen ein Hochschulstudium (z. B. Lörz und Schindler 2011) die hohen Kompetenzunterschiede erklären können, konnte anhand der hier analysierten Daten nicht geprüft werden, sollte aber zukünftig detaillierter untersucht werden.

Weiterhin lassen die Ergebnisse vermuten, dass sich zu Studienbeginn bestehende Kompetenzunterschiede zwischen den Fächergruppen im Verlauf des Studiums noch vergrößern (erklärte Leistungsvarianz: 4,6\% zu Studienbeginn vs. 7,6\% im sechsten Fachsemester). Zwischen den beiden leistungsstärksten bzw. -schwächsten Fächergruppen steigen diese von mittleren auf mittlere bis starke Effektstärken an (Studienbeginn: $0,49 \leq d \leq 0,58$; sechstes Fachsemester: $0,63 \leq d \leq 0,75$ ). Die Zunahme der Differenzen zwischen den Fächergruppen bleibt auch unter Kontrolle der Eingangsvoraussetzungen erhalten (siehe Tab. 6). Eine mögliche Erklärung für diese Vermutung liefern Unterschiede der digitalen Mediennutzung im Kontext fachkultureller Traditionen. Eine produktiv-reflexive Nutzung digitaler Medien (z. B. Massive Open Online Courses, interaktive Werkzeuge, kollaborative Lernformen), die mit einer Förderung der ICT Litercay einhergehen sollte, findet sich beispielsweise in der Informatik (Bestandteil der Ingenieurswissenschaften), die traditionell kreativaneignende Lernprozesse stark betont. In den Geistes- und Sozialwissenschaften dominiert hingegen eine eher rezeptive Nutzung konventionellerer ICT-Anwendungen (z.B. Weblogs, Textdokumente). Diese dienen vornehmlich der Begleitung und Dokumentation von Lernprozesse und unterstützen in geringerem Umfang die Weiterentwicklung digitaler Kompetenzen (Hofhues 2016; Persike und Friedrich 2016).

\subsection{Limitationen}

Mit der vorgestellten Studie konnten zwar neue Einblicke über die ICT Literacy angehender und fortgeschrittener Studierender generiert werden, jedoch sind - neben der erwähnten Frage der Eingangsselektivität - ebenso einschränkende Aspekte der Untersuchung zu berücksichtigen. Zum ersten beruht die vorliegende Arbeit auf Querschnittsdaten zweier unterschiedlicher Startkohorten, so dass die Überlegungen zu möglichen differenziellen Kompetenzentwicklungen in Abhängigkeit der Fächergruppe nur hypothetischen Charakter haben. Diese sind demnach an geeigneten Längsschnittdesigns - möglichst unter Einbezug der digitalen Mediennutzung in den jeweiligen Fächergruppen - empirisch zu prüfen.

Zum zweiten erfassen die statischen Aufgaben der NEPS-Tests nur ein Teilkonstrukt von ICT Literacy im Sinne einer wissensbasierten Kompetenz. Anhand von MC-Aufgaben werden zwar deklaratives Fakten- und Konzeptwissen sowie anwendungsorientierte Wissensbestände erfasst, aber die Anwendung erworbenen Wissens in realitätsnahen Situationen (prozedurales Handlungswissen), bleibt unberücksichtigt. Obwohl statische MC- und performanzbasierte ICT Literacy-Aufgaben substanziell miteinander ( $r \geq 0,70$; Senkbeil und Ihme 2014, in Vorbereitung) korrelieren und somit keine substanziell verschiedenen Befunde in Abhängigkeit vom Aufgabenformat zu erwarten sind, wäre eine genauere Prüfung der ICT Litercay an- 
hand performanzbasierer Aufgaben wünschenswert. Sie liefern umfangreichere, d. h. genauere diagnostische Informationen und könnten als Grundlage für Handlungsempfehlungen zur Sicherstellung grundlegender ICT-Kenntnisse und -Fertigkeiten von Studierenden genutzt werden.

Open Access Dieser Artikel wird unter der Creative Commons Namensnennung 4.0 International Lizenz (http://creativecommons.org/licenses/by/4.0/deed.de) veröffentlicht, welche die Nutzung, Vervielfältigung, Bearbeitung, Verbreitung und Wiedergabe in jeglichem Medium und Format erlaubt, sofern Sie den/die ursprünglichen Autor(en) und die Quelle ordnungsgemäß nennen, einen Link zur Creative Commons Lizenz beifügen und angeben, ob Änderungen vorgenommen wurden.

\section{Literatur}

Acemoglu, D., \& Autor, D.H. (2011). Skills, tasks and technologies: Implications for employment and earnings. Handbook of Labor Economics, 4, 1043-1171.

ACRL (2000). Information Literacy Competency Standards for Higher Education. San Antonio, Texas. http://www.ala.org/acrl/standards/. Zugegriffen: 11. Sept. 2018.

Becker, R., Haunberger, S., \& Schubert, F. (2010). Studienfachwahl als Spezialfall der Ausbildungsentscheidung und Berufswahl. Zeitschrift für ArbeitsmarktForschung, 42, 292-310.

Blignaut, A. S., \& Els, C. J. (2010). Comperacy assessment of postgraduate students' readiness for higher education. Internet and Higher Education, 13, 101-107.

Blömeke, S., Gustafsson, J.-E., \& Shavelson, R. J. (2015). Beyond dichotomies. Competence viewed as a continuum. Zeitschrift für Psychologie, 223, 3-13.

Blossfeld, H.-P., Roßbach, H.-G., \& von Maurice, J. (Hrsg.). (2011). Education as a lifelong process. The German National Educational Panel Study (NEPS) (Zeitschrift für Erziehungswissenschaft: Sonderheft 14). Wiesbaden: Springer VS.

Bos, W., Eickelmann, B., Gerick, J., Goldhammer, F., Schaumburg, H., Schwippert, K., Senkbeil, M., Schulz-Zander, R., \& Wendt, H. (Hrsg.). (2014). ICILS 2013 - Computer- und informationsbezogene Kompetenzen von Schülerinnen und Schülern in der 8. Jahrgangsstufe im internationalen Vergleich. Münster: Waxmann.

van Deursen, A. J. A. M., \& van Dijk, J. A. G. M. (2009). Improving digital skills for the use of online public information and services. Government Information Quarterly, 26, 333-340.

Eickelmann, B. (2017). Kompetenzen in der digitalen Welt. Konzepte und Perspektiven. Berlin: FriedrichEbert-Stiftung.

ETS (2002). Digital transformation. A framework for ICT literacy. Princeton: Educational Testing Service.

Ferrara, S., \& Lewis, D. M. (2012). The item-descriptor (ID) matching method. In G.J. Cizek (Hrsg.), Setting performance standards. Foundations, methods, and innovations (S. 255-282). New York: Routledge.

Goldman, S. R., Braasch, J.L.G., Wiley, J., Graesser, A.C., \& Brodowinska, K. (2012). Comprehending and learning from internet sources: Processing patterns of better and poorer learners. Reading Research Quarterly, 47, 356-381.

Haberkorn, K., \& Pohl, S. (2013). Cognitive basic skills - Data in the scientific use file. Bamberg: University of Bamberg.

Heinze, N. (2008). Bedarfsanalyse für das Projekt i-literacy: Empirische Untersuchung der Informationskompetenz der Studierenden der Universität Augsburg (Arbeitsbericht Nr. 19). Augsburg: Universität Augsburg.

Heinze, N., Fink, J., \& Wolf, S. (2009). Informationskompetenz und wissenschaftliches Arbeiten: Studienergebnisse und Empfehlungen zur wissenschaftlichen Recherche im Hochschulstudium (Arbeitsbericht Nr. 21). Augsburg: Universität Augsburg.

Hochschulforum Digitalisierung (2016). The Digital Turn - Hochschulbildung im digitalen Zeitalter. Essen: Edition Stifterverband - Verwaltungsgesellschaft für Wissenschaftspflege.

Hofhues, S. (2016). Informelles Lernen mit digitalen Medien in der Hochschule. In M. Rohs (Hrsg.), Handbuch Informelles Lernen (S. 529-546). Wiesbaden: Springer.

Hollingworth, S., Mansaray, A., Allen, K., \& Rose, A. (2011). Parents' perspectives on technology and children's learning in the home: social class and the role of habitus. Journal of Computer Assisted Learning, 27, 347-360. 
Huang, W.-H. D., Hood, D. W., \& Yoo, S. J. (2013). Gender divide and acceptance of collaborative web 2.0 applications for learning in higher education. Internet and Higher Education, 16, 57-65.

Katz, I. R. (2007). Testing information literacy in digital environments: ETS's iSkills assessment. Information Technology and Libraries, 26, 3-12.

Kleimann, B., Özkilic, M., \& Göcks, M. (2008). Studieren im Web 2.0. Studienbezogene Web- und E-Learning-Dienste. https://hisbus.his.de/hisbus/docs/hisbus21.pdf. Zugegriffen: 11. Sept. 2018.

KMK (2016). Bildung in der digitalen Welt. Strategie der Kultusministerkonferenz. Bonn: Kultusministerkonferenz.

Leichner, N., Peter, J., Mayer, A.-K., \& Krampen, G. (2014). Assessing information literacy programmes using information search tasks. Journal of Information Literacy, 8, 3-20.

Littlejohn, A., Beetham, H., \& McGill, L. (2012). Learning at the digital frontier: a review of digital literacies in theory and practice. Journal of Computer Assisted Learning, 28, 547-556.

Lörz, M., \& Schindler, S. (2011). Geschlechtsspezifische Unterschiede beim Übergang ins Studium. In A. Hadjar (Hrsg.), Geschlechtsspezifische Bildungsungleichheiten (S. 99-122). Wiesbaden: VS.

Mayer, A.-K., Leichner, N., \& Krampen, G. (2016). Förderung fachlicher Informationskompetenz von Psychologiestudierenden durch ein curricular integriertes Blended-Learning-Training? In M. Krämer, S. Preiser \& K. Brusdeylins (Hrsg.), Psychologiedidaktik und Evaluation XI (S. 193-201). Aachen: Shaker.

Nikolopoulou, K., \& Gialamas, V. (2011). Undergraduate students' information search practices. Themes in Science \& Technology Education, 4, 21-32.

Pant, H. A., Tiffin-Richards, S.P., \& Köller, O. (2010). Standard-Setting für Kompetenztests im LargeScale-Assessment (Zeitschrift für Pädagogik: 56. Beiheft, S. 175-188). Weinheim: Beltz.

Pensel, S., \& Hofhues, S. (2017). Digitale Lerninfrastrukturen an Hochschulen. Systematisches Review zu den Rahmenbedingungen für das Lehren und Lernen mit Medien an deutschen Hochschulen. http:// your-study.info/wp-content/uploads/2018/01/Review_Pensel_Hofhues.pdf. Zugegriffen: 11. Sept. 2018.

Persike, M., \& Friedrich, J. D. (2016). Lernen mit digitalen Medien aus Studierendenperspektive (Arbeitspapier Nr. 17). Berlin: Hochschulforum Digitalisierung.

Pohl, S., \& Carstensen, C.H. (2013). Scaling of competence tests in the National Educational Panel Study - Many questions, some answers, and further challenges. Journal for Educational Research Online, 5, 189-216.

Raish, V., \& Rimland, E. (2016). Employer perceptions of critical information literacy skills and digital badges. College and Research Libraries, 77, 87-113.

Rost, J. (2004). Lehrbuch Testtheorie - Testkonstruktion. Bern: Huber.

Rott, K. J. (2014). Medienkompetenz im Studium: Wie gut ist die Vorbereitung aufs Berufsfeld? In O. Zawacki-Richter, D. Kergel, N. Kleinefeld, P. Muckel, J. Stöter \& K. Brinkmann (Hrsg.), Teaching Trends 2014 (S. 153-170). Münster: Waxmann.

Rubin, D. B. (1987). Multiple imputation for nonresponse in surveys. New York: J.Wiley \& Sons.

Rutkowski, L., Gonzalez, E., Joncas, M., \& von Davier, M. (2010). International large-scale assessement data: Issues in secondary analysis and reporting. Educational Researcher, 39, 142-152.

Schmidt-Hertha, B., \& Rott, K. J. (2014). Developing media competence and work-related informational behavior in academic studies. International Journal on Advances in Education Research, 1, 90-108.

Schulmeister, R. (2009). Studierende, E-Learning und Web 2.0. In N. Apostolopoulos, H. Hoffmann, V. Mansmann \& A. Schwill (Hrsg.), E-Learning 2009. Lernen im digitalen Zeitalter (S. 129-140). Münster: Waxmann.

Schulmeister, R. (2010). Deconstructing the net generation theses. Qwerty - Open and Interdisciplinary Journal of Technology, Culture and Education, 5, 26-60.

Seeber, S., Nickolaus, R., Achtenhagen, F., Breuer, K., et al. (2010). Kompetenzdiagnostik in der Berufsbildung Begründung und Ausgestaltung eines Forschungsprogramms. Berufsbildung in Wissenschaft und Praxis, Beilage zu 1/2010. https://doi.org/10.2378/peu2017.art07.

Senkbeil, M. (2017). Profile computerbezogener Anreizfaktoren: Zusammenhänge mit ICT Literacy und sozialen Herkunftsmerkmalen. Ergebnisse aus der internationalen Schulleistungsstudie ICILS 2013. Psychologie in Erziehung und Unterricht, 64, 138-155.

Senkbeil, M., \& Ihme, J. M. Diagnostik von ICT Literacy: Messen Multiple-Choice-Aufgaben und simulationsbasierte Aufgaben vergleichbare Konstrukte? Vergleich der Testergebnisse zweier Instrumente aus den aktuellen Large-Scale-Studien ICILS 2013 und NEPS. Diagnostica. https://doi.org/10.1026/ 0012-1924/a000114.

Senkbeil, M., \& Ihme, J.M. (2014). Wie valide sind Papier-und-Bleistift-Tests zur Erfassung computerbezogener Kompetenzen? Diagnostica, 60, 22-34. 
Senkbeil, M., \& Ihme, J.M. (2017). NEPS technical report for computer literacy: scaling results of starting cohort 4 for grade 12 (NEPS Survey Paper No. 25). Bamberg: Leibniz Institute for Educational Trajectories, National Educational Panel Study.

Senkbeil, M., Ihme, J.M., \& Wittwer, J. (2013). Entwicklung und erste Validierung eines Tests zur Erfassung technologischer und informationsbezogener Literacy (TILT) für Jugendliche am Ende der Sekundarstufe I. Zeitschrift für Erziehungswissenschaft, 16, 671-691.

Smith, J. K., Given, L. M., Julien, H., Ouellette, D., \& DeLong, K. (2013). Information literacy proficiency: assessing the gap in high school students' readiness for undergraduate academic work. Library \& Information Science Research, 35, 88-96.

Sparks, J. R., Katz, I. R., \& Beile, P. M. (2016). Assessing digital information literacy in higher education: A review of existing frameworks and assessments with recommendations for next-generation assessment (Research Report. ETS RR-16-32. ETS Research Report Series).

Tannenbaum, R. J., \& Katz, I.R. (2008). Setting standards on the core and advanced iSkills assessments (Research Memorandum. ETS RM-08-04. ETS Research Report Series).

Teske, B., \& Etheridge, B. (2010). Information and communication technology literacy among first-year honors and non-honors Students: An assessment. Journal of the National Collegiate Honors Council—Online Archive, Paper 290. https://digitalcommons.unl.edu/nchcjournal/290. Zugegriffen: 04. November 2019.

Troboy, K., Batch, A.B., \& Bean, L. (2009). Information and communication technology assessment benchmark. AMCIS 2009 Proceedings, Paper 257. http://aisel.aisnet.org/cgi/viewcontent.cgi? article $=1263 \&$ context $=$ amcis2009. Zugegriffen: 27. Juni 2019.

Wallace, P., \& Clariana, R. B. (2005). Perception versus reality - Determining business students' computer literacy skills and need for instruction in information concepts and technology. Journal of Information Technolgy Education, 4, 141-150.

Walraven, A., Brand-Gruwel, S., \& Boshuizen, H.P. A. (2009). How students evaluate information and sources when searching the world wide web for information. Computers \& Education, 52, 234-246.

Wu, M.L., Adams, R. J., Wilson, M.R., \& Haldane, S. (2007). ACER ConQuest 2.0 - Generalised item response modeling software. Camberwell: ACER Press. 